\title{
III.
}

\section{Die Atomistik und Faradays Begriff der Materie.}

\author{
Eine logische Untersuchung \\ von
}

Otto Buek in Marburg.

\section{Prinzip und Hypothese.}

Das sachliche Verhältnis von Wissenschaft und Philosophie erscheint durch den geschichtlichen Hergang oftmals verdunkelt. Die Genies, in denen sich beide zu fruchtbarem Zusammenwirken verbinden, bilden die seltenen Ausnahmeerscheinungen, die den logischen Sachverhalt zu genauem und systematischem Ausdruck bringen. Der allgemeine wissenschaftliche Betrieb aber läßt die prinzipielle Klarheit hierüber nur zu häufig vermissen. Auch in der Gegenwart arbeiten wiederum Tendenzen in der Richtung einer Lockerung und Unabhängigmachung der Arbeit an den Prinzipien von der empirschen Forschung, einer Bestrebung, die ebenso verhängnisvoll für die W ssenschaft werden kann, wie für die Philosophie.

Je mehr der zu verarbeitende Stoff anwächst, je weitverzweigter die einzelnen Arbeitsgebiete sich gestalten, um so mehr glaubt man, die Prinzipien und die Theorie entbehren und sich der Feststellung des nackten Tatbestandes hingeben zu können. Demgegenüber meint wiederum die Philosophie die von der Wissenschaft verlassenen oder doch vernachlässigten Gebiete bebauen zu müssen, indem sie auf deren eigenstem Boden sich ansiedelt und die wissenschaftlichen Probleme mit eigenen und selbständigen Mitteln zu lösen versucht. Das ist der Abweg, der in die Irrungen der philosophischen Richtungen und Systeme auch in unserer Zeit hineingeführt hat. Daher muß streng und unerschütterlich an dem 
Gedanken festgehalten werden: da $B$ es eine doppelte Wahrheit nicht geben kann, in deren Bearbeitung die Philosophie und die Wissenschaft sich $z u$ teilen hätten. Bei der Trennung und Unterscheidung der Arbeitssphären kann es sich also lediglich um eine Unterscheidung des Standorts und des Gesichtspunkts handeln. Die Wissenschaft kann ihr Einheitsstreben nicht aufgeben, obne sich selbst und ihren Begriff aufzuheben. Es darf kein unerlaubter und unerhörter Gedanke bleiben, mit vollem Bewußtsein ein angestrebtes Ideal der Wissenschaft in den normierenden Grundlagen, mit denen sie operiert, zur Aufstellung zu bringen, ehe man zu dem Gebrauch dieser Grundlagen in der Ausfïhrung der Detailforschung schreitet. Die Wissenschaft hat Voraussetzungen zu ihrer Voraussetzung, von denen die wichtigste und erste diese ist, daß es Wissenschaft gebe, "scientiam esse“, wie der Erwecker des Idealismus, Nicolaus Cusanus, in der Morgenröte der Renaissance dem schlummernden Zeitalter zugerufen hat.

Dem Schein, daß es sich hierbei um eine bloße Tautologie oder um eine selbstverständliche Trivialität handle, muß mit aller Entschiedenheit entgegengearbeitet werden, wenn die Wissenschaft ihr eigenstes Problem: die Herstellung eines einheitlichen, logischen Zusammenhangs, einer geschlossenen, exakten Theorie nicht aus. den Augen verlieren soll, um sich zu einer trockenen und gedankenarmen Sammelarbeit von Kenntnissen zu veräußerlichen. Und andrerseits hat die Philosophie:das Problem, die Arbeitsweise, die Ergebnisse der empirischen Forschung in getreuem und gewissenhaftestem Sinne anzuerkennen und zu respektieren, will sie nicht jeden Zusammenhang mit der lebendigen Arbeit an der Kultur verlieren und ihren eigenen ungeordneten Träumen nachhängen. Von der $\dot{W}$ issenschaft selbst hat sie sich über ihr Problem und. ihre Methode belehren und instruieren zu lassen; nur so kann ibr ein eigenes Wirkungsgebiet und eine in ihren Schranken unangefochtene Selbständigkeit erwachsen und erhalten bleiben. Zum Glück läßt sich eine ernsthafte Ablösung der Wissenschaft von der Philosophie entgegen allen Bemühungen nicht durchführen. Je mehr sich die Wissenschaft von der Philosophie zu emanzipieren strebt, um so mehr sieht sie sich gezwungen, von deren Gedankengehalt in sich 
selbst aufzunehmen. Die Philosophie wird der Wissenschaft gleichsam einheimisch, d. h. sie erfüllt und durchdringt sie mit ihrem Geiste. Wo ernsthafte zusammenfassende wissenschaftliche Gedanken konzipiert werden, da regt sich immer von neuem das Bestreben, über die prinzipiellen Vorstellungen, die letzten bestimmenden Voraussetzungen vor sich selber Rechenschaft abzulegen. Wo die Bemühungen auf eine systematische Ordnung des Gesamtwissens ausgehen, da erhebt sich unausweichlich die Frage nach der maßgebenden Norm, die dem ganzen System und jedem Einzelinhalt in demselben ihren Wert und Charakter verleiht. Nie hat ein Denker, dessen Geistesarbeit sich nicht in der Einzelforschung verlor, dieser Frage, die eine logische und philosophische Frage $x . \tau^{2}$

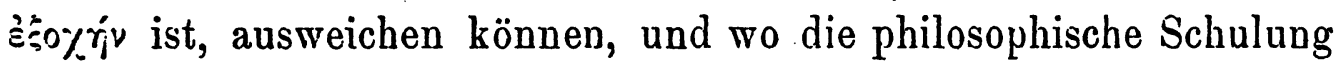
nicht zureichte, da hat er sie in eignen Zeichen und Symbolen auszudrücken und zu beantworten versucht. Aber freilich bleibt die Unzulänglichkeit des Ausdrucks stets mit einem begrifflichen Mangel behaftet. Mit einem stumpfen Werkzeug läßt sich kein Gedankenbild in scharfen Umrißlinien herausmeißeln. Um so dringlicher wird das Bedürfnis, solch' letzte Zeugnisse der Bedürftigkeit abzustoßen und die tief durchgreifenden wissenschaftlichen Vorstellungen der großen Forscher in W'orte einer philosophisch exakten Begriffssprache umzusetzen.

Der Umschwang und die Neubildungen unserer physikalischen Ausdrucksweisen stehen unter der starken und mächtigen Einwirkung der Gedankenwelt Faradays. Von ihm rührt der energische Anstoß her, der in unseren Tagen noch in raschem Siegeslauf die Experimentalphysik von Entdeckung zu Entdeckung führt, und man kann vielleicht sagen, daß die unzähligen Funde auf dem Gebiete der Elektrizität und des Magnetismus nur ebensoviele Bestätigungen der Faradarschen Grundanschauungen sind. Diese fortzeugende Irraft seiner Ansichten beruht in erster Linie auf der Fruchtbarkeit seirer systematischen Gedanken, seiner Methode, mit der er den Naturforschern ein Instrument in die Hand gab, dessen Anwendbarkeit sich in dem ihnen zugewiesenen Gebiet als nahezu unbeschränkt erwiesen hat. Nicht also im Einzelinhalt der von ihm hervorgebrachten Entdeckungen liegt ausschließlich die Bedeutung 
dieses Forschers, sondern an erster Stelle in der systembildenden Kraft seiner Anschauungen, und sie sind es, die seiner ganzen wissenschaftlichen Arbeit das Gepräge einer eminent philosophischen Leistung aufdrücken. Aus diesem Gesichtspunkt allein soll es hier versucht werden, das Werk Faradays zu verarbeiten, indem wir uns gleichsam in das Herz desselben versetzen und dort seine letzten Triebkräfte aufsuchen wollen. Freilich verlangt es unser Interesse, auch jenen geheimen Denkelementen nachzuspüren, die ihm selbst nicht immer ins Bewußtsein fielen, doch aber mit zwingender Notwendigkeit aus seinen Voraussetzungen folgen oder stillschweigend in ihnen enthalten sind. Auch wo er selbst den Faden fallen läßt, muß es erlaubt sein, ihm weiter nachzugehen und die Ergebnisse, zu denen er führt, mit.den Grundsätzen einer philosophischen Theorie zu vergleichen. Das historische Interesse ist auch hier mit dem sachlichen aufs engste verknüpft: wie weit nämlich die systematischen Anschauungen Faradays mit den Forderungen eines philosophisch fest gegründeten Systems vereinbar sind. Nehmen wir somit auch eine gewisse Freiheit in der Interpretation der leitenden Gedanken Faradays für uns in Anspruch, so wird es doch unsere Aufgabe sein, die Gründe und Anlässe dazu in seiner eignen Geistesarbeit nachzuweisen.

Faraday ist der große Meister des Experiments, und die gewaltigsten Entdeckungen auf dem Gebiete des Magnetismus und der Elektrizität sind mit seinem Namen verknüpft. „Kaum jemals hat ein einziger Mensch eine so große Reihe wissenschaftlicher Entdeckungen von folgenschwerster Bedeutung gemacht, wie Faraday", sagt Helmholtz. ${ }^{1}$, und er fährt fort: "Dabei waren fast alle diese Entdeckungen von der Art, daß sie auf unsre Vorstellungen von dem Wesen der Kräfte den eingreifendsten Einfluß ausübten"; wenngleich er ihm kurz vorher vorwirft, es sei ihm nicht gelungen, die ihn leitenden Gedankenverbindungen in klaren Worten wiederzugeben. In der Tat bietet das Studium des Faradayschen Werkes nicht geringe Schwierigkeiten dar. Denn weder ist es ihm vergönnt

1) Siehe Vorrede zu ,Tyndall, Faraday und seine Entdeckungen". Deutsch herausgegeben von H. Helmholtz, Braunschweig 1870. 
gerresen, in mathematischen Lauten zum Physiker zu reden, noch stand ihm für die Begründung und Formulierung der Prinzipien eine terminologisch exakte philosophische Sprache zur Verfügung. Die beiden großen Instrumente der Naturforschung: die Mathematik und die Philosophie waren ihm nicht als ererbtes Eigentum zugefallen, er mußte sie sich selbständig erarbeiten, auf mühevollen Umwegen sich ihren Besitz erkämpfen. Nit dem Scharfblick des Genies erkannte er aber bald ihren Wert und bediente sich ihrer nach seiner Art. Maxwell sagt von ihm: „Die Art, in welcher Faraday seine Kraftlinien benutzte, indem er die Phänomene der elektrischen Induktion beiordnete, beweisen, daß er ein Mathematiker ersten Ranges gewesen ist und einer, von dem die Mathematiker wertvolle und fruchtbare Methoden herleiten mögen, ${ }^{2}$ ) und Helmholtz (a. a. 0.) rühmt an ihm die philosophische Ader, die ilın an richtiger Stelle unter die vordersten in der allgemeinen wissenschaftlichen Gedankenarbeit unsres Zeitalters sich einreihen ließ. An diesem Zeugnis zweier hervorragender Forscher muß zuallererst die Anerkennung auffallen, daß der große Experimentator zugleich ein latenter Nathematiker und Philosoph gewesen ist. Die strenge Richtung auf die Phänomene und Tatsachen ließ ihn, also nicht untergehen in der Sammlung und Aufspeicherung derselben, sondern mit der empirischen Tatsachenforschung vereinigte sich der Drang nach logischer und methodischer Begründung und Ableitung der Naturerscheinungen. Wären uns nicht authentische Bestätigungen unsrer Ansicht überliefert, der Überblick über die gesamte Lebensarbeit und die Genesis dieser großen Reihen von Experimenten würde es bezeugen, daß ein idealistischer Zug durch das ganze Werk Faradays geht.

Die Tatsache ist ihm immer nur ein Anlaß zur Aufdeckung. und Feststellung des Gesetzes, die Konstatierung eines Phänomens: nur ein Schritt, eine Etappe, 'die auf die zugrundeliegende Gesetzlichkeit führt. Die Erscheinung in ihrer Isoliertheit absorbiert sein Interesse nicht, sondern auf die Ordnung und das Geset\% ist sein

2) Vgl. Mlichael Faraday von S. P. Tbompson. Deutsch von Schütte und Dannel. Balle 1900, p. 218 . 
ganzes Denken gerichtet. Gesetz und Tatsache sind immer in der methodischen Beziehung gedacht, die den Charakter der rationalen $W$ issenschaft ausmacht.

Jede Tatsache fordert das Gesetz heraus und jedes Geset\% enthüllt neue Tatsachen. Jedes neue Problem erzeugt neue Antworten und Lösungen, und jede Lösung gebiert neue Probleme. In dieser fortwirkenden Kraft der Methode entspringt die Genesis der Faradayschen Experimentaluntersuchungen, liegt der logische und idealistische Zug, der durch die Forschungen Faradays geht. Wer nach dem Gesetz, nach dem Prinzip fragt, der fragt nach der Vernunft; denn abgelöst von dem Wesen der Vernunft läßt sich kein Prinzip und kein Gesetz denken. Das Gesetz an den sinnlichen Stoff heranbringen, heißt den Gegenstand von der Gebundenheit an das einzelne, bestimmte Sein freimachen und ihn damit zu der Reinheit des Begriffes erheben. Indessen, wir haben auch glücklicherweise eine Reihe von direkten Äußerungen Faradays, die diesen Charakter seiner Wissenschaftsauffassung bestätigen und uns zugleich tiefer in die philosophischen Grundlagen seines Forschens einführen. Durch die ganze Lebensarbeit Faradays zieht sich eine unaufhörliche R.eflexion über die grundlegenden Begriffe der Physik und die Methoden, nach denen sie fortschreitet. Die Verfahrungsweisen der Wissenschaft, Hy pothese, Experiment, Theorie, ihre verschiedenen Geltungswerte, Prinzip, Tatsache und Gesetz, beschäftigen ihn fortwährend, und unermüdlich ist er bestrebt, den Anteil einer jeden Operationsmethode für die wissenschaftliche Forschung zu ermitteln und auszuwerten. Ihn verbindet ein verwandter Zug mit Newton in dem Widerwillen gegen die Hypothesen und gegen alles unbegründete und zweifelhafte Wissen. Indessen ist es bei ihm, der selbst beständig in.hypothetischen Vorstellungen sich erging, der selbst beständig Hypothesen erdachte und sie wieder fallen ließ, wenn sie sich als unbrauchbar erwiesen, nicht etwa prinzipielle Unklarheit über die Grundlagen der Wissenschaft, sondern vielmehr das Bestreben, die echten und fruchtbaren wissenschaftlichen Motive aufzusuchen und die Forschung von falschen und nicht begehbaren Pfaden abzulenken.

Wie Keppler den Tendenzausdruck „vera hypothesis", oder 
Newton den der „vera causa" prägt, so hält er den „Hypothesen“ und "Theorien" die Begriffe: „Tatsache“ und „Gesetz" entgegen. „Allein ich habe durchaus nicht die Absicht, auf derartige Betrachtungen oder auf die Bedeutung dieser Hypothese für die Theorie des Lichts und den hypothetischen Äther einzugehen. Mein Wunsch war vielmehr, gewisse Tatsachen der elektrischen Leitung und chemischen Verbindung beizubringen, welche eine große=Tragweite für unsere Ansichten über die Natur der Atome und der Materie haben, und so dazu beiżutragen, unser wirkliches Wissen im Gebiete der Naturwissenschaft, d. i. die Kenntnis der Tatsachen und Gesetze, von dem zu trennen, was zwar auch die Form eines Tissens hat, aber, da es so viel Hypothetisches in sich schließt, auch das grade Gegenteil davon sein kann". (Experimentaluntersuchungen über Elektrizität. Deutsch von Kalischer, Bd. II, S. 264.) An einer anderen Stelle warnt er: „Allein es ist immer sicher und philosophisch, Tatsache und Theorie soviel als möglich zu unterscheiden; die Erfahrungen vergangener Zeiten lehren uns genügend die Weisheit eines solchen Vorgehens; und wenn man bedenkt, daß der Geist die Neigung hat, sich bei einer Annahme zu beruhigen und, wenn sie den augenblicklichen $Z$ wecken genügt, zu vergessen, daß sie eben eine Annahme sei, so sollten wir dessen eingedenk sein, daß sie in solchen Fällen zu einem Vorurteil wird und unvermeidlich die Klarheit des Urteils mehr oder weniger trübt. Ich bezweifle, ob jemand, der als geschickter 'Theoretiker besonders befähigt ist, in die Geheimnisse der Natur zu dringen und die Art ihrer Tätigkeit durch Hypothesen zu erraten, auch, um zugleich sein eignes und anderer Fortschreiten zu einem zuverlässigen zu machen, besonders vorsichtig sein wird, das Wissen, welches aus Annahmen besteht, womit ich Theorie und Hypothese meine, von dem Wissen, welches Tatsachen und Gesetze umfaßt, zu unterscheiden, ob er weder je die ersteren zu der Würde oder dem Ansehen der letzteren erheben, noch die letzteren mehr, als unvermeidlich ist, mit den ersteren verwechseln wird" (a. a. 0. Bd. II p. 256 f.).

Und er lobt Newton und Arago: "Newton kann als Vorbild dienen, da er, wie aus seinen Briefen an Bentley hervorgeht, von 
der physischen Natur der Schwerkraftlinien zwar tief überzeugt, dennoch in seinen Veröffentlichungen bei dem Wirkungsgesetz der Kraft stehen blieb und hieraus seine großen Resultate herleitete; und ebenso Arago, der, als er die Erscheinungen des Rotationsmagnetismus entdeckte, und deren physikalische Ursache nicht erkannte, philosophisch genug dachte, um sich der Vermutungen zu enthalten " (a. a. 0. Bd. III. 485).

Es ist somit die durchaus "kritische Tendenz nicht za ver"kennen, über die Arten der Gewißheit, die in den Methoden der Wissenschaft liegen, sich Rechenschaft zu geben und sie in ihrer eigentümlichen Tragkraft und Leistungsfähigkeit zu unterscheiden. Theorie und Hypothese müssen etwas andres bedeuten als Tatsache und Gesetz: ihr selbständiger Geltungswert muß sorgsam unterschieden werden. Den Schutz und die Sicherung gegen vage Vermutungen und ihre Hypostasierung zum wahrhaften Sein, das ist es, was diese Unterscheidung besagen will. Den Zusammenhang mit den Phänomenen und der Wissenschaft, in der jene zur Bestimmung kommen, will die Berufung auf Gesetz und Tatsache ausdrücken; die durchgeführte Determination zum Naturgegenstand bedeutet die Korrelation von Gesetz und Tatsache, im Unterschied zu der nur versuchsweisen Setzung, zu der bloßen Disposition und Anlage zum Gegenstand, die Faraday mit den Ausdrücken Theorie und Hypothese bezeichnen will. Aber noch mehr soll diese Unterscheidung besagen. Ihr Sinn, der sich uns allerdings erst in anderem $\mathrm{Zu}$ sammenhange vollständig enthüllen wird, ist die Fernhaltung aller Versuche, nicht. in wissenschaftlicher Methodik sich der Naturdinge zu bemächtigen, sondern die Dinge selbst in einer Art zweiter Wirklichkeit zu hypostasieren. Es ist die Atomistik, an die die Mahnung ergeht, sofern sie, statt in Gesetzen des Denkens, in einer neuen Wëlt von Dingen die Wahrheit zu besitzen meint und so zum Vorurteil und Hemmschuh für einen gesetzmäßigen Fort: schritt wird. Nicht also in einem empiristischen Vorurteil, sondern in kritischer Reife ist die Bekämpfung der Hypothese durch Faraday gegründet, der den Wert der Hypothese wohl zu schätzen verstand. „Kein Forscher könnte ohne sie vorwärtsschreiten, und Bemühungen wie de la Rives, Ideen, die in ihrer früheren Gestalt einander ent- 
gegenstanden, in Harmonie zu bringen, sind von um so größerem Wert, als sie von einem Manne ausgehen, der ebenso den Wert der Hypothese, wie den der Gesetze, den Wert der Theorie ebenso wie den der Tatsachen kennt ..." (a. a. 0. Bd. III. p. 485).

Das Provozieren auf Gesetz und Tatsache gegen die Hypothesen stützt sich also nicht auf die dogmatische Annahme einer etwa schon im voraus gegebenen Welt, aus der dann durch Abstraktion das sie beherrschende Gesetz herausanalysiert werden soll, denn die Tatsache und das Gesetz sind für Faraday nichts Gegebenes oder Vorgefundenes, sie sind gar nicht der Ausgangspunkt für die wissenschaftliche Betrachtung, viel weniger deren Grundlage. Der wahre Ausgangspunkt, der sachliche Anfang liegt ganz wo anders, und es ist ein andrer Terminus, in dem er als Ausdruck dieses Sachverhalts zur Formulierung kommt. Das ist der Terminus des Prinzips, und es ist charakteristisch, wie an diesen Ausdruck, der an einer Stelle unzweideutig als logische Funktion gew ürdigt ${ }^{3}$ ) ist, die Kritik nicht ergeht, die gegen Theorie und Hypothese sich richtete. In Prinzipien muß der wahre Anfang der Wissenschaften gegründet werden, in letzten fundamentalen Grundsätzen muß sie ihren Ankergrund finden. Das Prinzip ist jedoch nicht etwa eine von den $\mathrm{Phänomenen}$ abgelesene oder aus den gegebenen Tatsachen herausavalysierte Wahrbeit, es ist der leitende Gedanke, der der Forschung den Weg weist. Das Prinzip gibt nicht nur das Mittel an die Hand, die gegebenen Erscheinungen in wissenschaftlichen Gesetzlichkeiten zu begründen, sondern es ist zugleich eine Anweisung zur Aufsuchung neuer Tatsachen und Gesetze, ein Schlüssel für neue Erscheinungen (Bd. III. p. 534 Art. 3363). Das Prinzip ist somit der höchste Gesichtspunktund die letzte Kontrollinstanz, dersich alle besondren Ideen über die Natur unterzuordnen haben. Jede physikalische Idee muß mit dem Prinzip verglichen, an ihm gemessen werden. Diese logische Rangordnung ist genau festzuhalten, wenn anders ein strenger Gang und eine feste Gesetzmäßigkeit in der Wissenschaft durchgeführt werden sollen. Indem Faraday das Gesetz von der Erhaltung der

3) Philosophical Jagazine Fol. XVII p. 169. 
Kraft als ein solches oberstes Prinzip einführt, sagt er weiter: "Agreeing with those, who admit the conservation of force to be a principle in physics as large and sure as that of the indestructibility of matter or the invariability of gravity, I think, that no particular idea of force has a right to unlimited or unqualified acceptance, that does not include assent to it." Ja die unbeschränkte Anwendung des Prinzips wird geradezu als Pflicht dem Denken zugemutet: "If the principle be accepted as true, we have a right to pursue it to its consequences, no matter what they may be. It is, indeed, a duty to do so. A theory may be perfectioned as far as it goes, but a consideration going beyond it is not for that reason to be shut out. We might as well accept our limited horizon as limit of the world. No magnitude either of the phaenomena or of the results to be dealt with should stop our exertions to ascertain by the use of the principle that something remains to be discovered and to trace in what direction that discovery may lie" (Philosophical Magazine vol. XIII. fourth series, 1857 p. 228).

Jetzt erkennen wir das innere Motiv der Bekämpfung der Hypothesen. Die Korrektur des Hypothesenbegriffs liegt im Prinzip. In streng gesetzmäßigem Gang soll sich der Fortschritt der Wissenschaft vollziehen. Die Garantie dafür aber liegt allein im Prinzip. Indem die wissenschaftliche Forschung durch Prinzipien weiterschreitet, erkennt sie über sich ein Gesetz an, vielmehr sie gibt es sich selber, damit aber schließt sie alle vagen und unmethodischen Vermutungen, die nicht im Zusammenhang mit dem gesetzmäßigen Gang der Wissenschaft stehen, von sich aus. Sofern die Hypothesen bloße unbegründete Annahmen, anschauliche Phantasiebilder sind, die nicht auf das Prinzip sich stützen, wird ihnen der Wert wissenschaftlicher Methoden abgesprochen. Damit ist jedoch die Hypothese nicht schlechthin als wissenschaftliches Mittel verworfen und entwertet, ihr Begriff .wird korrigiert in Rücksicht auf das Prinzip. In diesem mußseine letzte Grundlage haben, was eine rechte Hypothese sein will. So offenbart sich das Prinzip in seiner unerschöpflichen Fruchtbarkeit für die Erkenntnis; alle besonderen Ideen und Hypothesen erscheinen nunmehr nur noch als Selbstentfaltungen, als Speziali- 
sierungen der Prinzipien, d. i. der Methoden der Wissenschaft. Das ist die universale Bedeutung des Prinzips, das alle Gedanken über die Natur umspannt: die Natur selbst ist jetzt im Prinzip gegründet. Das Prinzip erscheint als das Universum, dem gegenüber jede besondere Theorie als der beschränkte Horizont des Menschen gilt. Im Lichte dieser Auffassung aber verrückt sich das Verhältnis von Hypothese und Tatsache, wie wir es früher kennen gelernt haben, sofern jetıt unter Hypothese nur der berichtigte Begriff einer reinen Grundlegung, d. i. einer nach dem Vorbild des Prinzips entworfenen, aus einer Hypothesis im Sinne Platons, oder einem reinen Grundsatz in der Sprache Kants abgeleiteten Voraussetzung verstanden werden muß. Einer solchen Hypothese gegenüber kann freilich die Tatsache nicht mehr als wissenschaftliche Instanz ausgespielt werden. Gegen das echte Prinzip verblaßt sie in ibrem Werte.

„No hypothesis should be admitted nor any assertion of a fact credited that denies the principle" (Philosoph. Mag. a. a. 0. 227); und an einer andern Stelle sagt er: „Da ich jedoch gerade hierüber rede, kann ich nicht umhin, den Wunsch auszusprechen, der eine und andre unter den Verteidigern der Kontakttheorie möge doch den Punkt in Erwägung ziehen, den man bisher sorgfältig umgangen zu haben scheint, daß nämlich die Annahme einer Kontaktkraft an sich ganz unphilosophisch ist. Eine Betrachtung dieser Art scheint mir der Kontakttheorie das Fundament selbst zu entziehen. Ich wünschte, Sie hielten es der Mühe wert, jene drei Seiten in Ihrem Magazin wieder abzudrucken. Nach meiner Auffassung drücken sie ein Fundamentalprinzip aus, das kein philosöphischer Kopf, dessen Denken selbst nur einen mäßigen Grad von Strenge besitzt, bei Seite schieben oder umgehen kann, und ich muß gestehen, so lange man sich über das Prinzip, das ich für ein Naturgesetz halte, nicht Rechenschaft ablegt, sei es auch nur der Schein einer solchen in Form einer Hypothese oder sonstwie, so lange werde ich sehr wenig Neigung verspüren, 'Tatsachen viel Wichtigkeit beizulegen, die zwar zu Gunsten der Kontakttheorie angeführt werden, die aber die Anhänger der chemischen Theorie stets ebenso für ihre ejgenen Ansichten sprechend und 
mit diesen im Einklang stehend finden" (Experimental-Untersuchungen Bd. II p. 248). Hiermit kehrt sich die Rangordnung um. Die Tatsache ist nicht mehr das Feste und Sichere, als welches sie der schlechten Hypothese gegenüber erschien, sondern gerade das Vieldeutigste und Schwankendste: ein bloß Relatives, das erst in der Beziehung auf das Prinzip seinen festen Wert erhält. In dieser Beziehung zu dem Prinzip bleibt sie dem Denken nicht mehr fremd, als dessen Ausdruck das Prinzip rekognosziert ist, sondern sie erscheint als eine Setzung des Denkens selbst, welche dieses kraft des Prinzips vollzieht. Die Tatsache erscheint als eine Antwort, die das Denken sich selbst gibt und die in der Sprache des Denkens erteilt werden muß. Daher ist auch nicht etwa die gegebene Wahrnehmung der letrte Zeuge für die Tatsächlichkeit, sondern sie muß es sich gefallen lassen, vom Denken und in Denken umgeschmolzen und umgeprägt zu werden durch das Experiment, in dem, vom Prinzip geleitet, die Vernunft an die Natur die Frage richtet und sie zwingt, auf dieselbe zu antworten.

In solcher Schärfe und Bestimmtheit ist freilich der Gedanke bei Faraday nirgends ausgesprochen. Noch immer, scheint es, bleibt für die Deutung Raum, als stände nicht sowohl die Tatsache als die Tats ächlichkeit unverrückbar fest und als bliebe dem Denken nur die nachträgliche Ausrechnung ihres Inhalts, die Auslegung ihres eigentlichen Sinnes und ihrer Bedeutung. Aber die Interpretation kann bei diesem Resultat nicht stehen. bleiben. Es ist ihre Aufgabe und ihr gutes Recht, den nicht zu Ende gedachten Gedanken in seine äußersten Konsequenzen und Ausläufer za verfolgen, den Ertrag der Untersuchung in seinen wichtigen Folgen für die Wissenschaft vorauszuberechnen und zu bestimmen. Unsere Interpretation.liegt in der Richtung des Faradayschen Gedankens. Die Feststellung der Tatsächlichkeit ist von der Erschließung ihrer Bedeutung und ihres Inhalts schlechterdings nicht zu trennen. Bedeutet die Tatsache nicht mehr als die bloße Forderung einer inhaltlichen Bestimmtheit, so ist sie als solche eben ein bloßes $\mathrm{X}$ und erscheint im Lichte des vollständigsten Gegensatzes zu ihrem eigentlichen Anspruch, eine absolute Bestimmtheit vertreten zu wollen. Will sie aber doch schon selbst einen Inhalt repräsentieren, 
so kann es nur dieser eine sein: nämlich ein schlechthin Einzelnes, ein Hier im Raum, ein Jetzt in der Zeit, womit sie sofort wieder der ganzen Gesetzlichkeit der Erkenntnis und dem Verfahren des Denkens anheimfällt. Jede einzelne räumliche und zeitliche Bestimmtheit ist unabtrennbar von den allgemeinen Erkenntnisbedingungen des Raumes und der Zeit und diesen gegenüber nicht das Frühere, sondern das Letzte. Erst das allgemeine Verfahren der Raumgesetzlichkeit liefert das Mittel zur Bestimmung des einzelnen, individuellen Raumpunktes. Daher führt der Gedanke Faradays in seinem Fortgang unausweichlich zu jenem wahren Verhältnis von Prinzip und Tatsache, wie es die idealistische Philosophie immer zum Ausdruck gebracht, und wie es in der praktischen Forscherarbeit Faradays eine überzeugende Ausprägung gefunden hat.

Alle Experimente Faradays sind von jenem Geiste geleitet, den Kant als die Revolution der Denkart, aus welcher die Physik als Wisseuschaft hervorgegangen ist, so schön beschreibt: ${ }^{4}$ ) „Als Galilei seine Kugeln die schiefe Fläcbe mit einer vom ihm selbst gewählten Schwere herabrollen, oder Torricelli die Luft ein Gewicht, was er sich zum voraus dem einer ihm bekannten Wassersäule gleich gedacht hatte, tragen ließ, oder in noch späterer Zeit Stahl Metalle in Kalk und diesen wiederum in Metall verwandelte, indem er ihnen etwas entzog und wiedergab, so ging allen Naturforschero ein Licht auf. Sie begriffen, daß die Vernunft nur das einsieht, was sie selbst nach ihrem Entwurfe hervorbringt, daß sie mit Prinzipien ihrer Urteile nach beständigen Gesetzen vorangehen und die Natur nötigen müsse, auf ihre Fragen zu antworten, nicht aber sich gleichsam am Leitbande gängeln lassen müsse; denn sonst hängen zufällige nach keinem vorher entworfenen Plane gemachte Beobachtungen gar nicht in einem notwendigen Gesetze zusammen, welches doch die Vernunft sucht."

Faradays Experimentaluntersuchungen bieten das Muster der von Kant geschilderten Methode. Vom Prinzip ausgehend und an seinem Faden fortgehend, baut er seine Hypothesen, die er, von Ex-

4) Krit. d. r. Vern. Vorländer p. 15. 
periment zu Experiment weiter schreitend, in immer engere Grenzen und Bestimmtheiten einschließt: mit dem Ziele der eindeutigen Determination des Gegenstandes, zum Naturobjekt. Das Gegebene ist im besten Falle nur die Frage oder das Problem, das an das Denken ergeht, und damit allerdings kein unverächtlicher Beitrag zur Erkenntnis; "aber andrerseits ist es ein ermutigender Gedanke, daß gerade sie es seien (d. h. die ungelösten Probleme), die, mit Eifer an der Hand des Versuches und mit Überlegung verfolgt, zu neuen Entdeckungen führen werden" (a. a. 0.246 Bd. II).

Ziel und Endzweck der Erkenntnis und der Wissenschaft, ihr Sein und ihre Realität aber ruht im Gesetz, das die Tatsachen umschließt, und in dem sie erst wahrhaftes Sein, Realität und Bestand haben. Das ist der große idealistische Zug, die echt philosophische Richtung der Faradayschen Physik. Es gibt kein Gesetz außer in und durch die Methoden des reinen Denkens. Die Natur ist dem Denken nicht fremd und der Geist ist kein. Spielball launischer Naturgewalten. Denn er selbst beherrscht und ordnet die widerstrebenden Krälte, indem er sie schöpferisch zur. Entdeckung bringt. Und es ist sicherlich keine Übertreibung, wenn wir hier die Ansicht aussprechen, daß diese Wahrheit des Idealismus für Faraday nicht ein leerer Schall,' sondern seine innerste wissenschaftliche Überzeugung war. Wie sie in seiner Forscherarbeit Wirklichkeit geworden ist, so hat er. sie, zwar nicht in philosophisch schulgerechter Form; aber doch nach seiner Art, in seiner Sprache, zum Ausdruck gebracht. Für diese Überzeugung hat er im Gefühl der Ehrfurcht und Bewunderang vor der Kraft der Vernunft noch in den letzten Jahren seines Lebens die schönen Worte gefunden: „Die Schönheit der'Elektrizität oder irgend einer andern Kraft besteht nicht in dem Geheimnisvollen und Unerwarteten, nicht darin, daß sie unversehens alle Sinne trifft, sondern darin, daß sie dem Gesetze unterworfen ist, und daß der gebildete Intellekt sie nunmehr im großen Naßstabe zu beherrschen vermag. Der menschliche Geist steht über, nicht unter ihr, und unter diesem Gesichtspunkte eben erscheint die Würde, die praktische Verwertung und der Nutzen der geistigen Ausbildung, welche die Naturwissenschaft gewährt, so überwältigend; denn indem sie den Geist befähigt, die 
Naturkräfte vermöge ihrer eigenen Gesetze in seinen Dienst zu stellen, überträgt sie göttliche Gaben auf den Menschen." (Exper. Unters. Bd. III p. 625).

\section{Die Atomistik.}

\section{Einleitung.}

Es ist wohl heute keine Frage mehr, daß die moderne Physik seit dem Zeitalter Newtons keine größere Umwälzung und Umgestaltung ihrer Grundlagen erfahren hat, als durch das Energieprinzip und die Faraday-Maxwellsche Theorie der Elektrizität. Bis auf Faraday galt das gesamte Streben der physikalischen Wissenschaft einer rein mechanischen Erklïrung der Naturerscheinungen; und diese Tendenz, die mit der Wriedergeburt der Wissenschaften im Zeitalter der Renaissance auftaucht, setzt sich bis in die heutige Zeit mit unverminderter Kraft und Lebendigkeit fort. Die mechanische Erklärungsart der Erscheinungen hat in der Atomtheorie eine ihrer typischsten Ausprägungen gefunden, und so ernsthaft die Atomistik seit den Tagen ihrer Entstehung von philosophischer wie ron wissenschaftlicher Seite bekämpft und angefeindet worden ist, sie hat sich bis auf die heutige Zeit, wie es scheint ungeschwächt, in ihrer Stellung behauptet. Aber wie die Entdeckung des Energiegesetzes, so ist Faradays Auftreten für die Entscheidung des Kampfes gegen den Atomismus eine neue und bedeutende Etappe. Indem er in den Streit eingreift, tritt die Kritik der Atomtheorie in ein neues Stadium. Denn diese Kritik ist nicht lediglich negativer Art; in der Yerteidigung gegen den erneuten Ansturm muß sich die atomistische Hypothese selbst eine Umbildung und Neugestaltung gefallen lassen, indem sie sich aus der Kritik mit neuen fruchtbaren Motiven bereichert und entwicklungsfähige Gedankenelemente in sich aufnimmt.

So sehr nun auch die Gegnerschaft liaradays gegen die Atomistik aus den unmittelbaren Erfahrungen und Problemen seiner eignen Tatsachenforschung entspringt, es verbindet sich bei ihm, wie überall so auch hier, mit dieser Tendenz ein eigentümliches prinzipielles Interesse. Seine Experimentaluntersuchungen bleiben 
immer an die Richtung seines Denkens auf die Herausarbeitung eines reinen und in sich abgeschlossenen wissenschaftlichen Systems aus dem zerstreuten Material der Einzelzusammenhänge gebunden. Und selbst wo diese Beziehung nicht offen am Tage liegt, läßt sich die enge Verkettung seiner Experimentaluntersuchungen mit seinen Anschauungen über die methodischen Grundlagen und fundamentalen wissenschaftlichen Prinzipien noch nachweisen. Sein Denken ist nicht vorzugsweise auf die Entdeckung und Ermittelung neuer Tatsachen und Gesetze gerichtet, sondern die Arbeit an den Problemen der Physik übt ihre rückwirkende Kraft auf die Begründung und Formulierung der letzten Voraussetzungen. Im Zusammenhang mit seinen Entdeckungen entsprangen ihm die auf die Revision der gangbaren physikalischen Anschauungen gerichteten Probleme, aber er versuchte es darum doch niemals, den Prinzipienstreit durch Appellation an die aus den nächstliegenden Bedürfnissen der Forschung erwachsenen Theorien und Hypothesen zu entscheiden. Vielmehr suchte er sich immer erst auch von der Zulässigkeit jeder besonderen hypothetischen Annahme zu überzeugen, durch Betrachtungen, die in seinen Voraussetzungen über den tiefsten methodischen Grund der Naturwissenschaft überhaupt wurzelten.

Die Tendenz seiner neuen Anschauung jst allerdings schon gleich im Beginn seiner wissenschaftlichen Laufbahn zu erkennen; es fehlt indessen nicht an Schwankungen und Zweifeln, über die er erst im. Laufe seiner Entwickelung Herr wird. An der Kritik und im Kampfe mit entgegengesetzten Auffassungen wächst ihm die Klarheit über den Sinn und die Bedeutung des von ihm neu eingeschlagenen Weges der Forschung. Schon früh konzipiert er den Gedanken, oder wie er es selbst auszudrücken liebt, „die Methode" der Kraftlinien, aber was ihm zuerst nur eine bequeme Operation zur Darstellung der gegebenen elektrischen oder magnetischen Krafterscheinungen ist, wächst sich zuletzt zu einer universellen Form der Wissenschaft aus, die den Anspruch erhebt, ein das gesamte Gebiet der physischen Kräfte umfassendes Ausdrucksmittel für die Wissenschaft zu werden. Dadurch gerät die neue Auffassung schon bald in Kollision mit der herrschenden Ansicht von den fernwirkenden Kräften, die sich dann in der Atomtheorie mit noch anderweitigen Voraussetzungen kom- 
pliziert. Und so wendet sich alsbald die Kritik gegen das Atom, an dessen Bekämpfung die Genesis der Motive zu der Konzeption der neuen Nethode der Kraftlinien sich deutlich verfolgen läßt.

\section{Die antike und die moderne Atomistik.}

Die Atomistik war im Zusammenhang der griechischen Spekulation über die Natur. der Materie entstanden. Gegenüber der Resignation des Eleatismus auf eine wissenschaftliche Erklärung der Naturerscheinungen und seinem Verharren im prinzipiellen Gegensatz zwischen dem geforderten Ideal des reinen Denkens und der Unzulänglichkeit aller sinnlich fundierten Erkenntnis, war die Atomistik mit dem Anspruch aufgetreten, diesen Gegensatz auszugleichen, den Widerspruch zwischen der Idee und den Phänomenen zu versöhnen.

Damit hatte zugleich das Problem der Erkenntnis eine neue Entwicklungsstufe erreicht; die Ideen des reinen Denkens wurden als Nethoden rekognosziert, die nicht nur fähig waren, wissenschaftlichen Inhalt zu erzengen, sondern die ihren Anspruch auf die Vertretung des wahren Seins nicht anders als in dieser Beziehung zur wissenschaftlichen Naturerklärung zu legitimieren vermochten. Die Atome und das Leere sollten jetzt nicht mehr eine vom Denken abgetrennte Wirklichkeit bedeuten, die unabhängig von den Problemen und Inhalten des Bewußtseins ihren Bestand hat, sondern sie wurden zu Erzeugnissen des reinen Denkens, zu methodischen Begriffen, mit denen das Bewußtsein Gesetz und. Ordnung an seinem Inhalte vollzieht, durch die es jenen Gegenstand hervorbringt, in dessen Gesetzlichkeit der Begriff der Natur besteht. In dieser Tendenz konnte Demokrit die Forschung der Eleaten mit dem Wort

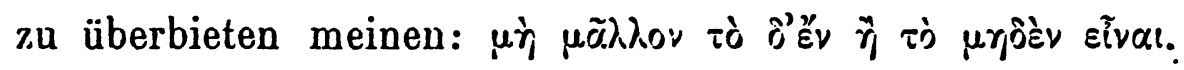

Wenn an das Atom als den Vertreter des Seins noch die Sinnlichkeit sich klammern mochte, so war das Leere als reine Gesetzlichkeit des Raumes von jeder Dinghaftigkeit frei und konnte nur in einer ewigen Idee, in einem reinen Verfahren des Denkens gegründet sein. Jarin lag der unverlierbare Gehalt der Atomistik, der sich auch an der Kritik des Sinnenscheins offenbart. Denn nur aus einem wethodischen Gesichtspunkt ist die Ablehnung der Sinnesqualitäten für den Aufbau der Erkenntnis zu verstehen. Wenn 
der Gegenstand der Natur den Bestand einer festen Gesetzlichkeit bedeuten will, so kann er nur in den Funktionen des Bewußtseins zur Bestimmung kommen, die einen solchen Bestand und eine solche sich identisch erhaltende Gesetzlichkeit zu erzeugen und zu verbürgen imstande sind. Die Sinne sind dazu schlechthin unvermögend; denn sie sind mit ihren wechselnden und verworrenen Zeugnissen die unzuverlässigsten und unbeständigsten Bürgen. Ein sich erhaltendes Sein vermögen sie nicht $z u$ gewährleisten. Darnach bleibt die alleinige Quelle für die Erkenntnis das reine Denken selbst, aus dem Gesetz und Ordnung fließt. Zahl, $\mathrm{Ma} B$, Gestalt und Lage werden als Begriffe des Denkens die einzigen Ausdrucksweisen für das wahrhafte Sein. Und es ist die Demokriteische Atomistik, die in diesen Begriffen die Natur. zu begründen

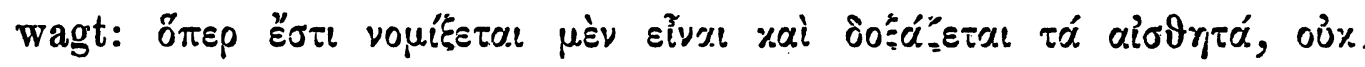

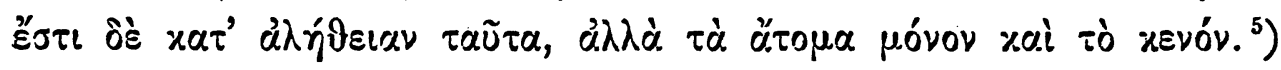

In den Atomen und dem Leeren allein die Wirklichkeit anbauen, heißt in Zahl, Maß, Gestalt und Lage die Erzeugung des Naturgegenstandes vollziehen. Die Natur wird damit zum Inbegriff einer mathematischen und mechanischen Gesetzlichkeit. Aber es scheint das Schicksal der Atomistik zu sein, daß sie im Verlauf ihrer Entwicklung mit all den Mängeln behaftet bleiben mußte, mit denen ihr erster unzulänglicher Ausdruck bei Demokrit kompliziert war. Ja, man kann sagen, daß sie sich über die methodische Schärfe und Klarheit, in der sie ihr Erfinder formulierte, seither nicht erhoben, sie. eigentlich nicht wieder erreicht hat. Schon bei ihrer Erneuerung durch Epikur hat sie einen Teil der charakteristisch Demokriteischen Züge eingebüßt. Wie ihre Begründung durch Epikur sensualistisch wird, so werden die von Demokrit entthronten subjektiven Sinnesqualitäten -wieder in ihr angemaßtes Recht eingesetzt. Nun wcrden sie dem wahrhaften Sein beigerechnet, als etwas Tatsächliches an einem gegebenen Gegenstande, der zu einer zweiten Wirklichkeit, neben der von außen in das Bewußtsein projizierten, erstarrt. ${ }^{6}$ ) Es ist charakteristisch, daß die Atomistik

5) Siehe Diels 406 nach Sextus Empiricus.

6) Vergl. Natorp; "Forschungen zur Geschichte des Erkenntnisproblems im Altertum", p. $223 \mathrm{ff}$. 
auch in ihren neueren Gestalten meistens mehr die Züge der Epikureischen als der Demokriteischen Fassung annimmt, wie sie denn auch der Lieblingsgedanke des Materialismus von jeher gewesen und geblieben ist, die Rüstkammer, der er seine besten Waffen entnehmen konnte. Es scheint der Atomistik somit die Tendenz beizuwohnen, im Dogmatismus zu verknöchern, und beinahe möchte man glauben, daß es das große Verdienst Demokrits war, vielmehr trotz der. Atomistik, als durch sie und mit ihrer: Hilfe, den idealistischen Grundzug seiner Lehre auszubilden.

Der Idealismus der atomistischen Lehre ist in der Erkenntnis des idealen Charakters der mathematischen Gesetze begründet und daher vermag er sich auch nur soweit zu erstrecken als die griechische Mathematik an ihrem Teil den Charakter der Zahlverhältnisse erkennen läßt. Daher ist auch der Atomismus eine notwendige Konsequenz der griechischen Zahlenlehre, wie er ja auch in der pythagoreischen ${ }^{7}$ ) Schule zuerst entworfen wird. Das Instrument der griechischen Mathematik ist die endliche Zahl, und so trägt auch der Atomismus die Signatur der diskreten Zahl bis auf den heutigen Tag. Wie das Verfahren mit den diskreten Zahlen ihre Gebilde durch Zusammensetzung fester und unbeweglicher Einheiten aufbaut, so ist das Atom das Element der Komposition, die diskrete physikalische Einheit, in deren Addition und Multiplikation die endlichen Dinge erwachsen. Und wie das Fundament der endlichen Zahlen die diskrete Einheit ist, so kann auch die atomistische Analyse nicht weiter hinabsteigen als bis zum diskreten Teil, der. vielleicht unter der Grenze der sinnlichen Anschaung liegt, immer doch aber innerbalb einer endlichen Ausdehnung beschlossen bleibt. Um der Unbestimmtheit, die in der willkürlichen Teilbarkeit ins Unendliche liegt, eine Schranke zu setzen, wird dem Atom eine absolute Starrheit beigelegt, wodurch es zugleich vor der Veränderung ${ }^{8}$ ) geschützt, und demnach sein Substanzcharakter verbürgt werden soll.

j) Siehe Cohen: Platons Ideenlehre und die Mathematik, p. 4.

8) Cnterdessen hat De Portu ("Galileis Begriff der Wissenschaft", Dürr, Leipzig 1904) an der traditionellen und auf Aristoteles zurückgehenden Auffassung des Demokriteischen Atombegriffs Zweifel erhoben. Die Atome brauchten nicht 
Das Gewaltsame dieser Voraussetzungen liegt im Charakter der endlichen Zahl, die das Kontinuum des Raumes aus sich nicht zu bestimmen vermag; ein Mangel, der sich auch in der Unbestimmtheit der Vorstellung von der Bewegung geltend macht. Die Bewegung ist als Ursache der Veränderung für die Komposition und Dekomposition der Atome gedacht, aber da auch sie erst als kontinuierliches Gebilde zur festen Bestimmtheit kommen kann, so fehlt auch dafür das Mittel, sie in ihrem Gesetz mathematisch ausdrückbar zu machen. Demgemäß müssen die Átomgestalten, die Zacken, Spitzen, Kanten u.s. w. das leisten, was die Bewegung nicht zu begründen vermag; die Verteilung und Gruppierung der substanziellen Individuen im Raume; und es kommt daher auch zu keiner klaren und genauen Ausbildung einer physikalischen Theorie, in der die Welt der Erscheinungen durch strenge Maßverhältnisse mit den Gesetzen des Denkens verknüpft wird und in ihnen Bestand gewinnt.

Für eine vorläufige Veranschaulichung und als ordnendes Prinzip dagegen reicht die Hypothese aus, und so erweist sie sich als hervorragendes Mittel zur Befriedigung des chemischen Interesses, indem das Interesse der Chemie in erster Linie gerichtet ist auf die Komposition der stofflichen Elemente in den zusammengesetzten Körpern und nicht auf den Prozeß des Überganges aus einem Zustand in den andern. Daher ist denn auch die Atomhypothese bis auf den heutigen Tag das wichtigste Erkenntnismittel in der wissenschaftlichen Chemie geblieben. Freilich hat sie sich sehr weit über ihre ursprüngliche Gestalt bei Demokrit und Epikur hinaus entwickeln müssen, um den modernen Anforderungen der Wissenschaft zu genügen. Der'gewaltige Aufschwung der Chemie in der néuesten Zeit erforderte präzisere Aufstellungen über die Beziehungen der Atome untereinander, und es ist in erster Linie die schärfere Fassung der quantitativen Verhältnisse und die Auf-

notwendig diskrete Einheiten zu sein, sondern könnten darnach als infinitesimale Elemente der Dinge gefaßt werden. Sollte diese Ansicht ihre historische Begründung finden, so wäre Demokrit von unseren Ausführungen, die sich allerdings auf eine bestimmte historische Form der Atomtheorie beziehen, auszunehmen. 
nahme mathematischer Bestimmtheit, welche durch die Verbindung mit den Bewegungsproblemen die Atomenlehre in den Stand setzten, mit Experiment und Beobachtung in Beziehung zu treten.

Nachdem die atomistische Theorie in der neuen Zeit durch Gassendi in ihrem Epikureischen Gewande wieder zum Leben erweckt worden war, wurde sie etwas später durch den großen Chemiker Robert Boyle als methodisches Werkzeug auch für die experimentierende Wissenschaft erschlossen. Sie legt damit ihre ausschließlich metaphysische Bedeutung $a b$, und wird $z u$ einem Prinzip für die wissenschaftliche Forschung. Aber freilich entwickelt und steigert sich ihre Fruchtbarkeit als wissenschaftliche Methode, je mehr es ihr gelingt, den Gehalt der physikalischen Prinzipien in sich aufzunehmen. Und je mehr sie sich mit dem physikalischen Inhalte erfüllt, umsomehr zieht, sie die Wissenschaft der Chemie selbst in die Interessen der Physik hinein. Die Atome Boyles sind noch ganz die vielgestaltigen Körperchen Epikurs, deren Zusammenhalt in nur sinnlicher Weise durch das Ineinandergreifen der Vorsprünge und Zacken erklärt wird. Aber der gewaltige Fortschritt der Physik, die Bewegungslehre Galileis und ihre Erweiterung durch Huyghens und Newton, lenken das Interesse immer mehr auf den Prozeß der Veränderung, auf den Übergang der Atome aus einer Lage in die andre, und erwecken das Verlangen, die Bewegung der Atome selbst in einem gesetzlichen Ausdruck zu erfassen.

Borelli und Huyghens gründen die Bewegungen der Atome auf die Gesetze des Stoßes, während die Atomistik durch Newton, gemäß seiner Theorie der fernwirkenden Kräfte, eine radikale Umbildung erfährt. Nunmehr beherrscht die Atomwelt dasselbe kosmische Gesetz der Schwere, das in den Bewegungen der Planeten sich betätigt, und die Konstellation der Atome wird organisiert durch die anziehenden und abstoßenden Kräfte, die in irgend einer Potenz der Entfernung wirken. Jetzt bedarf es auch der Berührung der Atome nicht mehr, da die Möglichkeit der Wechselwirkung im Gesetz der Fernkräfte ausreichend objektiviert ist. Auch die mannigfaltigen Atomgestalten verlieren ihre Funktion und werden, als überflüssig, fallen gelassen. In dieser wesentlich 
vereinfachten nud einheitlicher gestalteten Form orscheint die Atomistik im Beginn des 19. Jahrhunderts bei Dalton, Gay-Lussac und Avogadro, und wird nun zur herrschenden Methode in der modernen Chemie, die von da ab mächtig emporblüht. Die Theorie der Atomgewichte, die Molekulartheorie der Gase, die ganze organische Chemie ist das Gebiet, auf dem die neuere Atomistik sich in ihrer größten Fruchtbarkeit entfaltet, aber sie beginnt auch bald die Grenzgebiete der Chemie und Physik, die Thermo-Chemie und die. Elektro-Chemie zu umfassen, indem sie damit die Zurückführung der chemischen Erscheinungen auf die physikalischen Prinzipien anbahnt.

In diesem Stadium ihrer Entwicklung lernt Faraday die Atomtheorie kennen, gegen sie richtet sich in erster Linie sein Angriff und seine Kritik. In der Tat aber trifft diese Kritik nicht nur die Atomistik, die auf Newton zurückgeht, sondern ebensosehr die antike Atomistik, wie sie ihren Höhepunkt in Huyghens erreicht hat. Es ist somit eine bestimmte Gestalt der Atomtheorie, welche in ihren Motiven und Absichten von Faraday bekümpft und bestritten wird: nämlich der Atomismus antiker und Newtonscher Prägung. Wir werden sehen, wie Faraday den Gedanken des Atoms nicht schlechthin verwirft oder beseitigt, sondern selbst auf ihn zurückgreift, allerdings, um ihn von Grund aus umzugestalten. Hierbei werden wir freilich auf eine Schwierigkeit stoßen, welche die sonst so klare Sachlage zu trüben geeignet ist. Indem Faraday, wie schon oben erinnert wurde, den Begriff des Atoms in seine eigene Spekulation einführt, knüpft er an eine Fassung dieses Begriffes an, wie sie im 18. Jahrhundert von Boscovich gegeben, und in einem ganzen System der Naturphilosophie durchgeführt worden ist. Die Atomistik des Boscovich aber trägt ganz unverkennbar Newtonsche Züge an sich. Es wird daher nötig, die Anteile und Gedankenelemente, die von beiden Forschern in die Konzeption ihrer Grundbegriffe aufgenommen worden sind, scharf zu scheiden und zu sondern, wenn die wahre Absicht Faradays klar und deutlich hervortreten soll. Das wird um so notwendiger, als in neuerer und neuester Zeit mannigfache Versuche zu einer Neugestaltung und Neubelebung der.Atomhypothese gemacht worden sind, die 
sich auf Faradaysche Anschauungen stützen und eine Vereinbarung mit seinem Vorstellungskreis beabsichtigen. Daß sich die Argumente Faradays nicht ohne weiteres auch auf diese, ihm noch unbekannte Fassung beziehen, leuchtet von selbst ein. Wir werden daher an andrer Stelle noch von dem Verhältnis dieser neuen Theorien zum Faradayschen Begriffssystem zu sprechen und den Zusammenhang seiner Ideen mit einer verbesserten und reformierten Atomhypothese in Errvägung zu ziehen haben. Übrigens wirken aber die antiken und die älteren Newtonschen Atomtheorien bis in die jüngste Zeit hinein weiter, und immer von neuem ist es von physikalischer wie von philosophischer Seite unternommen worden, eine tiefere Begründung für eine Atomistik alten Stils, die wir hier zur Abkürzung als die diskrete gegenüber der Faradayschen, die wir mit Fechnei als kontinuierliche bezeichnen wollen, zu finden. Diese Versuche werden durch die Faradaysche Kritik mitbetroffen. Es muß also erlaubt sein, in einer vergleichenden Gegenüberstellung die Chancen der widerstreitenden Anschauungen für eine exakte Begründung der Naturwissenschaft gegeneinander abzuwägen, wobei denn freilich die einzelnen begrifflichen Elemente der verschiedenen Theorien gesondert und unterschieden voneinander behandelt werden müssen.

\section{Faradays Kritik.}

Die Opposition gegen die Atomtheorie regt sich bei Faraday schon sehr früh. In einer Untersuchung über die Kontaktwirkung des Platins sucht er sich über die Elastizität der Gase eine Vorstellung zu machen. Die atomistische Erklärung bietet sich ihm an, aber hier regt sich der Zweifel. „Wir haben nur sehr unvollkommene Vorstellungen von dem wirklichen und inneren Verhalten der Teilchen eines Körpers, der im festen, flüssigen und gasförmigen Zustand existiert, aber wenn wir auch, indem wir den gasförmigen Zustand von der gegenseitigen Abstoßung der Teilchen oder ihrer Atmosphären herleiten, am Ende im Irrtum sind, jedes Partikelchen als Kern einer Atmosphäre von Wärme, Elektrizität oder einem anderen Agens zu denken, so werden wir vermutlich doch nicht fehlgehen, wenn wir die Elastizität als auf Gegenseitigkeit der 
Wirkung beruhend betrachten" (Bd. I, Art. 626). Und in anderem Zusammenhang betont er nochmals das Lnsichere und Unbestimmte in der Vorstellung vom Atom. "Wiewohl wir von dem, was ein A tom sei, nichts wissen, so können wir doch nicht widerstehen, uns die Vorstellung von einem kleinen Teilchen zu bilden, welches uns dasselbe veranschaulicht, und wiewohl wir uns in derselben, wenn nicht in größerer Unkenntnis hinsichtlich der Elektrizität befinden, soda $\beta$ wir nicht imstande sind, zu sagen, ob sie eine besondere Materie sei, oder aus mehreren Materien bestehe, ob sie eine bloße Bewegung der gewöhnlichen Materie oder eine dritte Art Kraft oder Agens sei, so gibt es doch eine ungemein große Summe von Tatsachen, welche uns zur Annahme berechtigt, daß die Atome der Materie irgendwie begabt und vereinigt seien mit elektrischen Kräften, denen sie ihre hervorragendsten Eigenschaften und unter diesen ihre gegenseitige chemische Affinität verdanken". (Bd. I, p. 852.)

Schon hier also herrscht die Tendenz vor, den Begriff des Atoms gegen den der Kraft verschwinden zu lassen oder doch wenigstens auf die Seite zu stellen. Die Methode, die elektrischen Vorgänge in Linien elektrischer Kraft zu veranschaulichen, hat das Interesse am Atom verdrängt. Die Charakteristik des Atoms ist ja selbst erst möglich durch die ihm anhaftenden elektrischen Kräfte, die seine hervorragendsten Eigenschaften sind. Dieser Gedanke spitzt sich wenige Seiten weiter noch mehr ${ }^{\circ} z$.

„.. Die Äquivalentgewichte der Körper sind einfach diejenigen Mengen derselben, welche gleiche Mengen Elektrizität enthalten oder von Natur gleiche elektrische Krïfte besitzen. Es ist die Elektrizität, welche die Äquivalentzahl bedingt, weil sie die Verbindungskraft bedingt. .Aber wenn wir die Atomtheorie und deren Terminologie annehmen, so sind es die in ihrer gewöhnlichen chemischen Aktion einander äquivalenten Atome der Körper, welche von Natur mit gleichen Mengen Elektrizität vereinigt sind. Aber ich muß gestehen, ich bin mißtrauisch gegen den Ausdruck Atom; denn es ist sehr leicht vọ Atomen zu reden; aber sehr schwer, sich eine klare Vorstellung von ihrer Natur zu bilden, insbesondre wenn zusammengesetzte . Körper in Betracht kommen." (Bd. I, Art. p. 869.) 
Hier scheint somit die atomistische Veranschaulichung des Vorganges geradezu als eine überflüssige Annahme aufgefaßt zu sein, eine unnötige Verwickelung und Komplizierung, welche die Vorstellung von dem wirklichen Tatbestand eher verdunkelt als schärfer und präziser macht. Das Verhältnis der elektrischen und chemischen Kräfte scheint auszureichen zur Darstellung des elektrolytischen Gesetzes. Die neuen und speziellen Annahmen, welche die Atomtheorie über den bloßen Ausdruck der Gesetze hinaus von sich aus hinzubringt, lassen die Atomistik als untauglich erscheinen, ein Prinzip der Naturforschung zu sein. Denn indem die Atomtheorie ihre Voraussetzungen durch eine Reihe von komplizierten und besondren Hypothesen belastet, verliert sie den Charakter einer letzten unableitbaren Grundlegung des Denkens und nimmt die Farbe eines sinnlich gegebenen Tatbestandes an. „Die Atomtheorie wird in unsrer Zeit auf diese oder jene Weise vielfach angewandt, namentlich zur Erklärung der Erscheinungen der Kristallisation und der Chemie, aber man unterscheidet sie nicht mit solcher Sorgfalt von Tatsachen, daß sie nicht dem Lernenden als ein Ausdruck der Tatsachen selbst erscheint, wiewohl sie im besten Falle nur eine Hypothese ist, deren Wahrheit wir nicht dartun können, was wir auch ron ihrer Wahrscheiulichkeit sagen oder denken mögen. Mit dem Worte „Atom“, welches niemals gebraucht werden kann, ohne viel gänzlich Hypothetisches in sich zu schließen, beabsichtigt man oft eine einfache Tatsache auszudrücken, aber so gut auch die Absicht sein mag, so habe ich doch niemanden gefunden, der dasselbe ron den es begleitenden verführerischen Vorstellungen frei zu halten vermocht hätte, und es unterliegt wohl keinem Zweifel, daß Ausdrücke wie feste Proportionen, Äquivalente, Urbestandteile und so weiter, welche alle Tatsachen der sogenannten Atomtheorie in der Chemie vollständig ausdrückten, und auch jetzt noch ausdrücken, deshalb aufgegeben wurden, weil sie nicht bezeichnend genug waren, um alles das auszusagen, was derjenige, welcher dafür das Wort "Atom" gebrauchte, sich dabei dachte; sie drückten nicht sowohl die Hypothese, als die Tatsache aus." (Bd. II, Seite 256.)

Das Gefährliche und Verführerische, das an der Vorstellung von den Atomen haftet, liegt in dem Bestreben, für die rein gesetzlichen 
Relationen ein anschauliches Bild nach Art der Sinnendinge zu gewinnen. Es ist die versteckte Rücksicht auf die Sinnlichkeit, die, indem sie nach einem Ausdruck für die physischen Vorgänge sucht, die Vorstellung der Atome erzeugt. Die Entdeckung, daß die chemischen Verbindungen nach bestimmten einfachen Zahlenverhältnissen stattfinden, erhält in der quantitativen Feststellung der Äquivalentgewichte ihre ausreichenđe Objektivierung. In dem zahlenmäßigen Ausdruck der chemischen Äquivalente liegt. für Faraday schon die genügende Bürgschaft für die Tatsächlichkeit der Verwandtschaftsbeziehungen. Der deutsche Chemiker Richter, der Mitentdecker des Gesetzes der multipeln Proportionen, schloß ähnlich wie Faraday. Die mathematische Formulierung des Gesetzes genügte ihm, gemäß seiner Anschauung, daß sich die Vorgänge der Natur nach Maß, Zahl und Gewicht vollziehen. In den idealen Relationen der Mathematik schien die Tatsache selbst genügend gefestigt zu sein. Dalton beruhigte sich bei dem Gedanken nicht. Er fühlte sich erst befriedigt, als er die Äquivalentgewichte als Folgerung aus der atomistischen Vorstellung der chemischen Vorgänge ableiten konnte. Ist die Verbindung ein Zusammentreten von Atomen, so war die Proportionalität gemäß einfachen rationalen Zahlen eine einleuchtende, auch sinnlich evident zu machende Vorstellung. Auch die Gewichtsverschiedenheit der einzelnen Verbindungen ließ sich durch die Zahl der Atome und ihr spezifisches Gewicht ausreichend erklären und vorstellig machen.

So bequem und erfolgreich jedoch ein solches Verfahren sein mag, es liegt die Gefahr darin, die eine Aufnahme sinnlicher Bestandteile in die Voraussetzungen der Wissenschaft mit sich bringt. Alle sinnlichen Vorstellungen enthalten in sich ein Moment der Unbestimmtheit und Willkürlichkeit und verführen dadurch zu Annahmen, die sich wissenschaftlich nicht kontrollieren lassen. Wo aber das methodische Kriterium und die Kontrolle an den Gesetzen der wissenschaftlichen Logik verloren geht, da hat die Phantasie freien Spielraum und es entsteht die Gefahr, daß die Grenzen der Wissenschaft und metaphysischen Phantasie ineinanderlaufen. Wo andrerseits der Geist in der sinnlichen Evidenz zum Stillstand kommt, da beruhigt er sich leicht und schnell bei einer gegebenen 
Erklärung und mit dem methodischen Leitgedanken geht der Trieb zur Revision und Korrektur, zum Hinausschreiten über jede Stufe der Erkenntnis, und damit der Fortschritt der Wissenschaft verloren.

Daher kommt die Atomistik schnell zu letzten Annahmen, die die Fragen des Denkens einfach abschneiden, und sie verfällt in den Fehler, letzte Qualitäten zu postulieren, über die sie die Rechenschaft verweigert. Es ist dies ein Mangel der Atomistik, den besonders tief Leibniz erkannt und in einem Brief an Hartsoeker gerïgt hat. „Diese ursprünglichen Qualitäten sind für gewöhnlich die Zufluchtsstätten entweder für die hinter einem schönen Namen sich versteckende Faulheit, weil man es liebt, die Erforschung einzuschränken und sich mit geringeren Kosten aus der Sache zu ziehen, oder endlich für das Vertrauen, mit dem man sich selbst bewundert, daß man den Dingen auf den Grund gekommen ist, wohingegen die Natur, welche die Unendlichkeit ihres Urhebers ausdrückt, niemals diese Arten von Schranken annimmt, bei denen unser Geist stillzustehen sucht." Tatsächlich ist die Tendenz in der Atomistik nicht zu verkennen, sie von ihrer methodischen Aufgabe loszulösen und zum Ausdruck einer absoluten Wirklichkeit zu verselbständigen. In der Messung der absoluten Größen der Atome, ihrer Abstände etc. spricht sich diese Tendenz der Atomistik aus. Das Atom ist nicht mehr das Atom der Synthese, aus der die Gegenstände aufgebaut werden, sondern bloß das Atom der Analyse. Damit aber wird es selbst zu einem Ding, das einer empirischen Messung unterliegt; es wird selbst zum Problem, das einer Lösung harrt, die doch wohl nicht wieder durch atomistische Voraussetzungen $z u$ bewerkstelligen sein kann, ohne dem Verdacht einer tautologischen Erklärung oder einer Zurückschiebung ins Unendliche zu verfallen.

Aber auch die willkürliche Hypothesenbildung begünstigt, ja fordert die Atomistik, da ihre.große Unbestimmtheit eine Konkretisierung ihrer Annahmen verlangt, die sie nicht aus ihren eignen Mitteln zu leisten vermag. Indem von vornherein über die Gestalt, Größe, Entfernung und Geschwindigkeit der Atome nichts ausgemacht ist, läßt sich durch die willkürliche Variierung und Festsetzung dè einzelnen Parameter eine gleichermaßen berechtigte und zwingende 
Ableitung der 'Tatsachen erzielen. Und wenn freilich durch eine immer größere Anzahl von Daten sich der Kreis der möglichen Hypothesen immer mehr verengt, so darf andrerseits auch nicht vergessen werden, daß mit dem Bekanntwerden neuer Daten auch die Chancen der Atomtheorie überhaupt gegen die anderer Naturerklärungen abnehmen.

So glaubte Faraday schon zu seiner Zeit mit seiner Methode der Kraftlinien bei einer geringeren Anzahl von hypothetischen Voraussetzungen eine größere Präzision erreichen zu können, und er bekämpft die Atomtheorie wegen der großen Zahl besonderer Annahmen, die sie nötig hat: „In der großen Mannigfaltigkeit dieser Hypothesen und ihrer raschen Aufeinanderfolge scheint mir mehr der Beweis der Schwäche als Strenge dieses Gebietes der Physik zu liegen, und man muß die breiten Annahmen, die einer jeden zugrunde gelegt sind, stets gegenwärtig haben. Selbst in der vollkommensten derselben, der von de la Rive, sind diese Annahmen beträchtlich. Denn man muß sich die Molekel, wie groß auch die Anzahl der Atome sein mag, aus denen jedes einzelne besteht, als flache oder scheibenförmige Körper deuken; man muß annehmen, daß die Atome des einen Molekels nicht die Anordnung der Atome eines anderen stören oder durchbrechen; da $\beta$ die elektrochemischen Vorgänge mit einer solchen Konstitution der Molekel in Einklang stehen können, daß die bewegende Kraft eines jeden Atomstromes ihren Sitz in der Achse hat und daß andrerseits dem Durchgang des Stromes durch die Oberfläche sich Widerstand darbietet u.s. w." (Bd. III, p. 484.)

\section{Fechners Einwand.}

Man.könnte gegenüber diesen Einwänden versucht sein, doch auf die Erfolge hinzuweisen, welche die Atomistik bisher innerhalb der Physik und besonders der Chemie zu erringen vermochte. Fechner hat in einer ausführlichen Darstellung, auf die wir noch später zurüickkommen, es versucht, aus einem solchen Gesichtspunkte die Atomtheorie nicht nur zu verteidigen, sondern selbst als notwendige Voraussetzung der Wissenschaft hinzustellen.

Er sagt: „Statt dessen stellt sich die Sache vielmehr so: Läßt 
sich statt durch strenge Rücksichtnahme auf selbst noch untereinander streitige Ansichten und Voraussetzungen, wie das die philosophischen faktisch sind, durch Rücksicht auf einen Zusammenhang unbestritten feststehender Tatsachen zeigen, daß der Atomismus feststeht, so ist eben damit bewiesen, daß die philosophischen Grundlagen, mit denen er nicht bestehen kann, selbst nicht bestehen können und alle jene philosophischen Betrachtungsweisen, die ihn einstimmig verurteilen, sich hiermit.selbst verurteilen" (Atomenlehre II. Aufl. p. 16); und ferner: „Wir werden, kurz gesagt, zu zeigen suchen, daß die Atomistik richtig ist, weil sie für die Wissenschaft des Faktischen notwendig ist, und nur das faktisch Richtige kann für die Wissenschaft des Faktischen notwendig sein" (Seite 5).

Dagegen kann bemerkt werden, daß dieser Einwand auf dem Standpunkt Faradays bereits antiquiert ist, der nicht oft genug erinnern konnte, Theorie und Gesetze, Prinzip und Tatsache zu unterscheiden. Für Fechner ist die Atomistik, die das Tatsächliche begrïnden und erklären soll, selbst zu einem laktum, einem faktisch Richtigen geworden, wie er denn auch ihre Gewißheit, oder, was er so nennt, mit der Wahrscheinlichkeit der Umdrehung der Erde um die Sonne vergleicht. Und wenn er im folgenden mit einer ziemlich dürftigen Reihe von physikalischen Erscheinungen, deren Erklärung angeblich nur durch die Atomtheorie zu leisten sei, die dynamische Theorie der Materie zu erledigen versucht, so vermögen wir ihm auf diesem Wege nicht zu folgen. Für uns handelt es sich nicht um die Frage, welche physikalische Theorie uns instandsetzt, eine möglichst große Anzahl von Erscheinungen bequem zusammenzufassen und technisch $\mathrm{zu}$ beherrschen, sondern wir versuchen, einen widerspruchsfreien und mit sich einstimmigen Begriff der Natur, einen Begriff der Wissenschaft, zu finden, welcher den strengen Anforderungen des Denkens genügt, aus dem erst eine wahre und philosophisch korrekte Definition der Tatsachen gewonnen werden kann. Den aber kann uns keine Einzelheit liefern, und jedem Versuch in dieser Richtung möchten wir mit den schon erwähnten Worten Faradays begegnen: „So lange man sich über das Prinzip, das ich für ein Naturgesetz halte, nicht Rechenschaft abgelegt hat, so lange werde ich wenig Neigung spüren, Tatsachen viel Wichtig- 
keit beizulegen, die zwar \%ugunsten der Kontakttheorie angeführt werden, die aber die Anhïnger der chemischen Theorie stets ebenso für ihre eignen Ansichten sprechend, mit diesen in Einklang stehend finden" (Bd. II p. 249).

Das Verfehlen des Problems durch Fechner enthüllt sich zudem sehr bald. Er sagt: „Denn die der Physik wie jeder Wissenschaft immanente Philosophie besteht nicht darin, daß sie von einem gewissen Punkte ihres Gebietes an die ihr eigentümlichen allgemeinen Kategorien und Methoden fallen lasse und auf die Philosophie überspringe, sondern daß sie die ihr wesentlichen bis ins Letzte durchbilde“ (Atoml. p. 46). Diese Durchbildung der Kategorien „bis ins Letzte" ist es nun aber gerade, was wir allein als Aufgabe der Philosophie anerkennen. Darüber hinaus bestehen für uns keine Probleme, die einer philosophischen Bearbeitung unterlägen, aber gerade diese. den Wissenschaften immanente Philosophie, wie Fechner sie nennt, und die strenge Durchführung ihrer Methoden „bis ins Letzte“ verbietet den Begriff des Atoms. Es ist gerade umgekehrt, wie Fechner es will, wenn er sagt: „Sie (scil. die Atomistik) mag durch die Philosophie draußen verworfen werden, aber sie wird durch die Philosophie drinnen gefordert" (p.46). Das ist es eben, was bestritten wird. Die Philosophie "draußen" ist kein zulässiges Problem. Die Philosophie „drinnen“ aber widersetzt sich der atomistischen Weltansicht. Und auch nur aus solchen inneren, der Wissenschaft selbst immanenten Motiven bekämpft Faraday die Atomistik. Seine Kritik bezieht sich auf den Atomismus lediglich, sofern er Wissenschaft sein, „Erfahrung ermöglichen" will, wobei er freilich unter Erfahrung nicht eine Summe von Wahrnehmungen, und unter Wissenschaft eine bequeme Handhabe und Technik des Gedächtnisses zur Aufbewahrung dieser Wahrnehmungen versteht, sondern ein nach Prinzipien des Denkens, nach strengen Gesetzen zusammenhängendes System von Erkenntnissen. Ein solches System vermag der Atomismus nicht herzustellen, weil er die Gesetzlichkeit des Denkens verleugnet, in welcher alle Wissenschaft gegründet liegt. Die Musterung der Kategorien des reinen Denkens und ihre Durchführung "bis ins Letzte" führt zu dieser Ansicht über das Atom. 
5. Die Kontinuität des Denkens und das Atom.

Der Absolutismus des Atombegriffs hindert diese Durchführung: des Atoms. Das Abbrechen der Teilbarkeit an einem bestimmten Punkte hat von jeher Schwierigkeiten gemacht, und es ist eine physikalische Bestimmtheit, welche die Diskretheit der Partikel legitimieren muß. Das Atom muß sich die Prädikation der absoluten Hürte gefallen lassen, um sich in seiner konstanten, beharrlichen Ausgedehntheit zu erhalten. Und wie die Endlichkeit des Volumens das Problem nur weiter hinunter ins Unsinnliche verlegt, ohne es für den Gedanken aufzuheben, so verlețt die absolute Härte das Gesetz der Kontinuität. Die absolute Härte bedeutet eine unendliche Kraft des Widerstandes, eine unendliche Fraft aber ist kein Objekt möglicher Erfahrung, kein in einer Quantität realisierbarer Begriff. Das Unendliche bedeutet die Gesetzlichkeit des Denkprozesses, des Verfahrens einẹr Setzung, das in keiner endlichen Setzung zur Ruhe kommt. Der Gegenstand der Natur aber verlangt Bestimmtheit und Determiniertheit. Die Annahme einer absoluten Widerstandskraft für das endliche Atom würde somit einer Durchbrechung des Kontinuitätsgesetzes gleichkommen. Deun von den in endlichen Quanten auszudrückenden Kräften der Natur zu der in keiner endlichen Setzung ausdrückbaren Widerstandskraft gäbe es keinen Übergang.

In dieser Tendenz ist das Wort Faradays zu verstehen: „Einen graduellen Unterschied, ja selbst einen Unterschied in der Natur der Kraft, der mit dem Kontinuitätsgesetz verträglich ist, kann ich zugeben, aber einen Unterschied zwischen einem kleinen, harten Teilchen und den es umgebenden Kräften kann ich mir nicht denken" (Bd. II p. 261). Es ist hier derselbe Gedanke leitend, den Leibniz in zahlreichen Wiederholungen in seinem Briefwechsel mit Huyghens und Hartsoeker ins Feld führt. Auch für ihn liegt der Anstoß in der Verletzung des Kontinuitätsgesetzes, welche die Annahme einer absoluten Härte für die Atome zur Folge hat. „Die große Beständigkeit der Natur gibt durchaus keine Veranlassung $z u$ dem Glauben an die Atome“, so schreibt er an Hartsoeker, „sondern sie. stößt diesen im Gegenteil um. Sie wirkt in den kleinsten Teilen wie in den großen, es 
verhält sich dort alles genau so wie hier. Wenn wir Augen hätten, die zu dicsem \%wecke scharfsichtig genug wären, würden wir sehen, daff auch dio kleinsten 'l'eilo zerbrechen und einander zerquetschen kïunen, und es ist hierin gar kein Grund für einen unendlich großen Widerstand vorhanden. " Er nennt es „zu einem Wunder oder einer qualitas occulta seine Zuflucht nehmen", wenn man den Grund der Verhinder'ung woiterer 'T'eilbarkeit in letyten T'eilen der Materie sucht. „Die Atome sind die Wirkung der Schwäche unserer sinnlichen Anschauung, die es liebt sich auszuruhen und sich zu beeilen, um bei dem Immerweiterteilen oder den Analysen zu einem Abschluß zu gelangen; nicht so verhält es sich in der Natur, die vom Unendlichen ausgeht und zum Unendlichen hinstrebt. Daher genügen die Atome nur der sinnlichen Anschauung, aber mit den höheren Gründen stehen sie im Widerspruch."

Es ist charakteristisch, daß sich in diesem Gedanken Faraday mit den klassischen Denkern der Philosophie zusammenfindet. An diesem Punkte kommt er dem Idealismus mit Bewußtsein ganz nahe, und es ist bezeichnend, daß, indem er sich vom Gedanken der Kontinuität und durchgängigen Gesetzmäßigkeit der Erscheinungen ergriffen fühlt, die Ahnung in ihn aufsteigt, daß diese Universalität aus dem Denken stammen möchte: „Sie (die Naturwissenschaft) lehrt uns Kleines und $G r o ß e s$ stets miteinander zu vergleichen und zwar unter nahezu unendlich verschiedenen Bedingungen, denn das Kleine umfaßt im Prinzip ebenso oft das Große, als dieses das Kleine, und so wird das Jenken umfassend. Sie lehrt uns, die Prinzipien mit Sorgfalt zu deduzieren, sie festzuhalten oder das Urteil noch aufzuschieben, die Gesetze zu entdecken und ihnen zu gehorchen und durch sie ermutigt $z u$ werden, das, was wir von dem Kleinsten wissen, auch auf das Größte anzuwenden." Es ist wie ein Gruß, der aus einem anderen klassischen Zeitalter der Wissenschaft zu uns herüberklingt. Anaxagoras dürfte der erste sein, der das Motiv der uneingeschränliten und unerschöpflichen Souveränität der Denkgesetzlichkeit zur Anerkennung und auf die unendliche Teilbarkeit der Materie

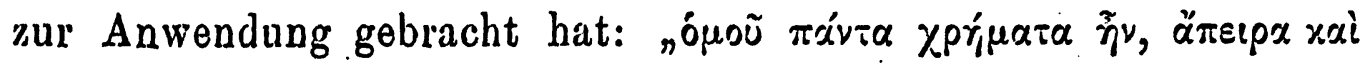

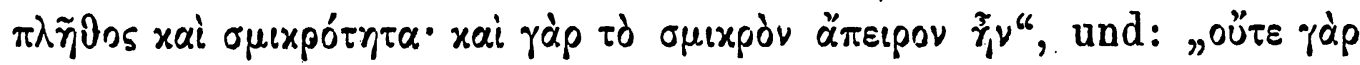

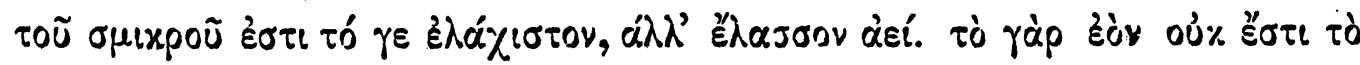




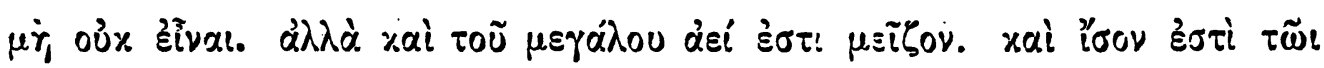

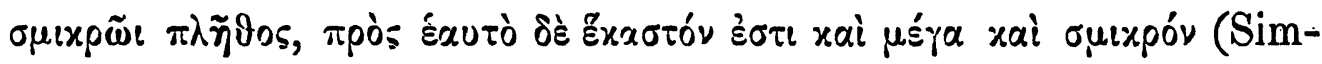
plic. phys.155,23 ff. und 155,30 s. Diels „Die Fragmente der Vorsokratiker" p. 326 und 327).

Man vergleiche damit, wie Malebranche denselben Gedanken einmal zum Ausdruck bringt: Tant il est vrai que l'esprit voit l'infini aussi bien dans le petit que dans le grand: non par la division ou la multiplication réitérées de ses idées finies, qui ne pourraient jamais atteindre à l'infini, mais par l'infinité même qu'il découvre dans ses idées et qui leur appartient, lesquelles lui apprennent tout d'un coup, d'une part qu'il n'y a point d'unité, et de l'autre point de bornes dans l'étendue intelligible (Entretiens sur la métaphysique éd. Jules Simon, p. 14). Die einzelne Setzung kann keine Schranke für die Erkenntnis bedeuten wollen, in der sie sich gleichsam verdichtet und erstarrt. Die Einheit behält ihre Festigkeit und Umschriebenheit unter einem bestimmten, fixen Gesichtspunkt. Nichts aber nötigt das Denken, in diesem Gesichtspunkt zu verharren, sondern der Prozeß der Erkenntnis treibt selbst zu einem beständigen Wechsel des Winkels, unter dem wir die Erscheinungen betrachten. Dagegen sind die Atome in letzter Instanz endliche Körperchen. Für das Denken aber gibt es keine absolute Größe oder Kleinheit. Das Verfahren der quantitativen Setzung ist nicht bedingt oder eingeschränkt durch die Einheit, mit welcher sie operiert, für sie ist das kleinste Atom in nichts unterschieden von den Dimensionen eines Planeten oder einer Zentralsonne, und es ist schlechthin Willkür, bei einer bestimmten Ausdehnung stehen zu bleiben, solange nicht das Verfahren der Teilbarkeit in einem positiven Gesichtspunkt seine Begrenzung findet. Jeder Punkt, an dem die Teilbarkeit abgeschnitten wird, bleibt eine unbegründete Setzung, ja die Atomistik läßt sie in letzter Rücksicht überhaupt unbestimmt. Sie verfällt damit dem Vorwurf, okkulte Qualitäten und Erdichtungen statt Gründen einzuführen. „Erdichtungen zu machen ist leicht, schwer aber ist es, sio vernünftig zu machen, $d$. h. zu zeigen, daß es solches gibt oder $\mathrm{da} ß$ zum renigsten ein Grund für sie vorhanden ist. Die Atome sind solch eine Erdichtung; ein vollkommen flüssiges erstes Element 
ist eine zwoite. Die vollkommene Flüssigkeit ist ebenso widervernünftig wie die vollkommene Ilärte. In der Natur gibt es nichts derartiges, statt dessen ist in ihr eine unendliche Anzahl von Widerstandsgraden vorhanden, sodaß jeder Körper mit Bezug auf andere und vergleichsweise einen Grad von Flüssigkeit und einen Grad von Festigkeit hat. ${ }^{49}$ )

Die Natur ist eben kein dem Bewußtsein koordiniertes Ding, mit dem das Denken fertig worden soll, wie es mag, sondern ein in den idealen Verfahrungsweisen zu erzeugendes Gebilde des Gedankens; dann aber muß sie eben den Konstruktionsgesetzen des Bewußtseins sich fügen und aus ihnen entspringen. Das Verfahren des Denkens ist ein Setzen von Relationen und Beziehungen und kein Abbilden fertiger und konstanter Wesenheiten. Und wie in der idealen Relation der Glieder der Gegenstand entsteht und besteht, so ist jeder Punkt, bei dem das Denken Halt macht, nur ein vorläufiger Ruhepunkt, den es unter einem bestimmten Gesichtspunkt setzt und festhält, keine unübersteigbare Schranke, an der es zum Stillstand kommen muß. Das Gesetz des Denkens vermag, oder ist vielmehr genötigt, jeden solchen Punkt wieder in einer neuen Relationssetzung aufzuheben, jede vergleichsweise Station wieder zu überschreiten. Soll der Gegenstand der Natur sich auflösen in ein reines Erzeugnis des Bewußtseins, so muß er den Bedingungen der reinen Denkmethoden unterworfen bleiben. Es ist nicht zulässig, daß, was für die eine Voraussetzung gilt, in der anderen wieder aufgehoben werde. Die Methoden des reinen Denkens müssen sich durchdringen und determinieren, nicht aber sich bekämpfen und stören. Die Geometrie ist eine der wichtigsten Bedingungen zum Aufbau des Gegenstandes der Natur. Was in ihr als griundlegende Bedingung für den Gegenstand der Physik sich herausgestellt hat, darf nachträglich nicht in einer neuen Setzung wieder über den Haufen geworfen werden. ${ }^{10}$ ) Die mathema-

9) Leibniz an Härtsoeker (Leibniz, Philosophische Scbriften, hrsg. v. Gerhardt. . Bd. III, p. 506).

10) Der Fehler, den die heutigen Anḅänger der Atomistik begehen, liegt in letztem Grunde darin, daß sie sich in ihren physikalischen Begriffen noch nicht dem fortgeschrittenen bistorischen Stand der modernen Mathe- 
tischen Gesetze, sofern sie als Erkenntnisfaktoren deduziert worden sind, müssen mit derselben Strenge wie in der Geometrie für die Physik in Geltung bleiben, mit Rücksicht auf welche sie ja einzig zur Definition gekommen sind. Das Atom ist zu verwerfen, weil es diese Ordnung und dieses Abhängigkeitsverhältnis ignoriert und umstößt. Von Descartes rührt eine Formulierung des Widerspruches her, der den Atombegriff als logisches Unding unmöglich macht, und der von diesem Punkte aus besonders deutlich erkennbar wird. „Ainsi nous pouuous dire qu'il implicque contradiction, qu'il y ait des atomes, ou des parties de matiere qui ayent de l'extension et toutefois qui soient indiuisebles, à cause qu'on ne peut auoir l'idée d'une chose estenduë, qu'on ne puisse auoir aussy celle de sa moitié, ou de son tiers, ny, par consequent, sans qu'on la conçoiue diuisible en 2 ou en 3. Car de cela seul que je considere les deux moitiez d'une partie de matiere, tant petite qu'elle puisse estre, comme deux substances completes, et quarum ideae non redduntur a me inadaequatae per abstractionem intellectus, je conclus certainement qu'elles sont reellement diuisibles. Et si on me disoit que, nonobstant que je les puisse conceuoir, je ne sçay pas, pour cela, si Dieu ne les a point unies ou jointes ensemble d'un lien si estroit, qu'elles soient entierement inseparables, et ainsy que je n'ay pas raison de le nier; je repondrois que, de quelque lieu qu'il puisse les auoir jointes, je suis assuré qu'il peut aussy les deioindre de façon qu'absolument parlant, j'ay raison de les nommer diuisibles, puis qu'il ma donné la faculté de les conceuoir comme telles". ${ }^{11}$ )

Aus derselben Einsicht fordert Kant die unendliche Teilbarkeit auch als Methode für die Physik: ${ }^{12}$ ) "Wider diesen Satz einer unendlichen Teilung der Materie, dessen Beweisgrund bloß mathematisch ist, werden von den Monadisten ${ }^{13}$ ) Einwürfe vor-

matik akkonodiert haben. Die antike Atomistik ist, wie oben ausgefübrt, aus der Jathematik der Alten entsprungen. Die Entdeckung der Analysis des Unendlichen erledigte daber auch den Grundgedanken des antiken Atomismus.

11) Correspondance, éd. Adam et Tannery. Paris 1899. Bd. 111, p. $477 \mathrm{f}$.

12) Kant, Kritik der reinen Vernunft (Vorländer, p. 387).

13) Kants Vorwurf trifft natürlich den Urbeber des Monadenbegriffs nicht, den er selbst von diesem Urteil ausschließt. In der Sache selbst seben wir 
gebracht, welche sich dadurch schon verdächtig machen, daß sie die klarsten mathematischen Beweise nicht für Einsichten in die Beschaffenheit des Raumes, sofern er in der Tat die formale Bedingung der Möglichkeit aller Materie ist, wollen gelten lassen, sondern sie nur als Schlüsse aus abstrakten aber willkürlichen Begriffen ansehen, die auf wirkliche Dinge nicht bezogen werden könnten. Gleich als wenn es auch nur möglich wäre, eine andere Art der Anschauung zu erdenken, als die in der ursprünglichen Anschauung des Raumes gegeben wird, und die Bestimmungen desselben a priori nicht zugleich alles dasjenige beträfen, was dadurch allein möglich ist, $\mathrm{da} B$ es diesen Raum erfüllt." Und in derselben Tendenz postuliert Leibniz die allgemeine Teilbarkeit: „Es war bereits", wie Cassirer a. a. 0. p. 347 schreibt, „ausgesprochen, daß die aktuellen Dinge sich von den idealen Regeln der Mathematik nicht entfernen können und daß hierin die Realität besteht, die Erscheinungen von Träumen scheidet. Dem Prinzip der unendlichen Teilung ist als einer notwendigen Grundlage der Mathematik die uneingeschränkte Anwendbarkeit auf die phänomenale Wirklichkeit verbürgt. Die Schranke, die man am Problem des physischen Punktes zwischen Geometrie und Physik aufzurichten sucht, muß fallen; ihre Behauptung gründet sich auf eine falsche Fragestellung im Begriff der Realität. »Je ne conçois point d'indivisibles physiques et je crois que la nature peut exécuter toute la petitesse que la Géometrie peut considérer" ".

Ist somit die unendliche Teilbarkeit auch innerhalb der Physik aus methodischen Gründen gefordert; so muß damit die Vorstellung der absoluten Härte der ausgedehnten Atome fallen, und die Konsequenz führt vielmehr zu der Annahme einer schlechthin elastischen Materie, die eine unbeschränkte Differenzierung der Teile bis ins Unendliche zuläßt. Und wie Leibniz, so zieht Faraday diese notwendige Konsequenz: „Anstatt, die Atome als überaus hart. und unveränderlich in ihrer Form anzusehen, kann man sie sich als höchst elastisch vorstellen; schon das Zusammendrücken einer

ihn in völliger Einmütigkeit mit Leibniz, vergl. Kritik der reinen Vernunft, p. 388; Cassirer, Leibniz' System in seinen wissenschaftlichen Grundlagen. Marburg 1902, p. 348. 
Iuftblase mit den Händen kann ihre Größe ein wenig ändern und in dem Versuche von Cagniard de la Tour wird diese Änderung in der Größe so weit getrieben, daß die Volumina sich um das Vielhundertfache unterscheiden. Ebenso ist es, wenn ein fester oder flüssiger Körper sich in Dampf verwandelt" (Exp. Unts. Bd. II, 262).

\section{Substanz und Atom.}

Wenn aber die unendliche Teilbarkeit auch für die physikalischen Körper proklamiert ist, so verliert die Vorstellung des Atoms ihren Sinn, oder sie muß sich eine radikale Umbildung gefallen lassen, die mit ihren wichtigsten Voraussetzungen aufräumt. Die Grenze, welcher die Teilung der Materie zustrebt, ist der unausgedehnte Punkt; statt dessen postuliert das Atom das letzte beharrliche und unveränderliche Element in einem Partikel von bestimmter Ausdehnung und Gestalt. In der Tat scheint das Prinzip der Substanz ein solches letztes unveränderliches Substrat zu erfordern und das Atom in der Festhaltung eines solchen identisch sich Erhaltenden diese Forderung zu realisieren. Und im räumlichen Nebeneinander allein, denkt man, kann es gelingen, den Fluß des Geschehens zum Stehen zu bringen. So hatte Descartes die Ausdehnung zur Substanz gemacht und einer der Atomistik verwandten aber kontinuierlichen Korpuskularphysik gehuldigt.

Indessen, ist nicht in der Setzung des Korpuskels, des ausgedehnten Atoms, das Beharrliche vielmehr vorweggenommen als zur Erzeugung gebracht? Die Substanz als reine Funktion darf nicht in einem fertigen Ergebnis antizipiert, sondern muß im Prozeß der Erkenntnis ibren Trsprung finden. Die räumliche Ausdehnung dagegen ist immer schon Ergebnis, immer schon ein Ende und kein eigentlicher Anfang. Darum darf sie nicht einfach hypostasiert werden; sonst ist und bleibt sie eine Erdichtung nach dem Ausdrucke Leibnizens. Gegen diese Hypostasierung der ausgedehnten Partikel richtet sich die Kritik, die in weiterer Verfolgung des Gedankens 'gegen das Atom ergeht. Ind indem Faraday seine Neigung zur Annahme von Kraftzentren bekennt, beruft er sich auf den Jesuiten Boscovich, der mit einer eigentümlichen atomistischen Theorie um die Mitte des 18. Jahrhunderts hervorgetreten war. Wenn man schon An- 
nahmen machen wolle, so sei offentsar der sicherste Weg, so wenig Annahmen als möglich zu machen, und in dieser Hinsicht scheine ihm Boscovichs Atombegriff einen großen Vorzug vor dem gewöhnlichen zu haben. "Seine Atome sind, “ fährt er fort, „wenn ich es recht verstehe, bloße Kraftzentren, nicht materielle Teilchen, in denen die Kräfte selbst ihren Sitz haben. Wenn wir das materielle Teilchen der gewöhnlichen Atomtheorie ohne seine Kräfte $a$, und das System von Kräften in ihm und um das Teilchen $m$ nennen, so verschwindet in Boskovichs Theorie $a$ oder "es ist blos ein mathematischer Punkt, während es nach der gewöhnlichen Vorstellung ein unveränderliches, undurchdringliches Stückchen Materie und $m$ eine es umgebende Kraftatmosphäre ist" (a. a. 0. p. 260, Bd. II).

Er exemplifiziert seine Auffassung an dem Beispiel der elektrischen Leitung: "Kehren wir beispielsweise zum Kalium zurück, in welchem Metall, wie wir gesehen haben, der gewöhnlichen Theorie zufolge, die Atome sehr weit voneinander abstehen müssen, wie können wir uns einen Augenblick denken, daß sein Leitungsvermögen anderswoher stamme, als aus den Eigenschaften des Raumes oder nach meiner obigen Bezeichnung von $m$ ? Ebenso müssen auch seine anderen Eigenschaften hinsichtlich des Lichts, des Magnetismus, der Starrheit, der Härte, des spezifischen Gewichtes, eine Folge der Eigenschaften oder Kräfte von $m$ sein und nicht der von $a$, das vielmehr ohne die Kräfte, als eigenschaftslos, aufgefaßt wird. Aber dann ist sicherlich $\dot{m}$ selbst die Materie des. Kaliums; denn wo gäbe es auch nur den mindesten Grund (wenn man nicht willkürliche Annahmen machen will), einen qualitativen Unterschied zu machen zwischen dem Raume in der Mitte zwischen den Zentren zweier unmittelbar benächbarter Atome und irgend einer anderen Stelle zwischen diesen Zentren ... . Für mich verschwindet also der Kern $a$ und die Substanz besteht in den Kräften $m$. In der Tat, welche Vorstellung können wir uns denn von dem Kern, getrennt von seinen Kräften, bilden? Unsere ganze Auffassung und unser Wịsen: vom Atom, ja selbst unsere Einbildungskraft ist beschränkt auf dẹn Bégriff von seinen Kräften. Was bleibt denn für unser Denken übrig; woran wị die Vorstellung von einem $a$ :ge- 
trennt von den anerkanntermaßen existierenden Kräften knüpfen könnten? Es mag ja dem, der über diesen Gegenstand zum ersten Male nachdenkt, schwer werden sich die Kräfte der Materie unabhängig von einem für sich existierenden Etwas, das man , die

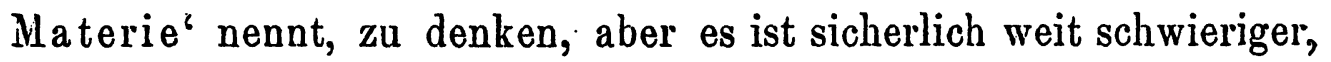
ja tatsächlich unmöglich, sich die Materie unabhängig von den Kräften zu denken oder vorzustellen. Nun kennen wir die Kräfte und anerkennen ihr Dasein in jeglicher Erscheinung der Welt, die abstrakte Materie dagegen in keiner; warum. nehmen wir denn das Dasein von Etwas an, von dem wir doch nichts wissen, das wir uns nicht vorstellen können, und wozu auch keine theoretische Notwendigkeit vorhanden ist?" (Exp. Unters. Bd. II, p. 261.) Hier sehen wir nun die schon früh auftauchende Tendenz, dem Kraftbegriff gegenüber dem der Materie die logische Priorität zuzuerkennen, zu vollster Klarheit gereift. Die Materie ist eine Vorstellung, die sich nicht zu begrifflicher Klarheit und Schärfe bringen läßt. Tnd mit bewunderungswürdiger Bestimmtheit werden die Anteile unterschieden, welche die methodischen Ansprüche des Denkens und die Aufforderungen der Sinnlichkeit am Begriff der Materie haben. Die Kraft erscheint als das Bekannte und Vertraute, als das Gegebene, gegenüber dem Kern $a$, der bloßen Ausdehnung, die als eine besondere physische Realität von der Kraftatmosphäre unterschieden wird.

Die „abstrakte Materie" heißt nun der Kern, der als ein bloßes räumliches oder existierendes „Etwas“ dem Dasein der Kräfte angehängt wird. In der Tat, was ist dieser Kern, der die Materie repräsentieren soll, wenn er mehr sein will als das Ausdehnungsund Herrschaftsgebiet der Kräfte. Fällt er mit dem Kraftbereiche selbst zusammen, so ist er nichts als der geometrische Raum, losgelöst von seinem dynamischen Inhalt, ein bloßes Abstraktum, eine reine Erkenntnisbedingung, die auf Grund einer neuen selbständigen Setzung doppelt gesetzt ist. Soll er aber doch mehr sein, so ist er nur die Idee einer Existenz, die bloße Forderung eines Seins, die erst in den Kräften ibre Befriedigung und Realisierung finden kann; auch in diesem Falle eine reine Verdoppelung des einen Faktors, der schon einmal in den Kräften selbst in Anrechnung gekommen ist. 
Es ist immer wieder die T'üuschung des Absoluten, die nicht anders als in einem abgetrennten, der Welt der Wandlungen und Vcränderungen entzogenen Sein ihren Ankergrund zu finden vermeint, die alte absolute Substanz, die im atomistischen Materiebegriff sich noch inmitten der Wissenschaft einen Platz gesichert hat. Ihre Entlarvung bedeutet zugleich ihre Entthronung und Vertreibung aus dem Reiche der exakten, wissenschaftlichen Arbeit. Die Substanz ist für die Wissenschaft da, und diese hat es einzig und allein mit der Welt der Phänomene zu tun.'

Die phänomenale Welt ist jedoch die Welt der Wandelbarkeit und der Veränderung. Die Substanz muß in das Verhältnis zu diesen Veränderungen eingehen, wie sie selbst eine Relation, ein Verbältnis ist. Und innerhalb dieses Verhältnisses zur Veränderung hat sie ihre Funktion zu vollziehen, ihre Aufgabe zu vollenden. Sie steht in der unaufhebbaren Korrelation zu den Inhärenzen, die den eigentlichen Inhalt und die Fülle des Seins in der Erscheinung bezeichnen. Sie selbst wird uns nur in ihren Inhärenzen bekannt, welche ihrerseits durch sie zur Erkenntnis und Bestimmung kommen sollen. Die Veränderungen aber werden durch Kräfte gemessen; und an den Kräften und in Bezug auf sie allein kann die Substanz in Funktion treten. Für sich selbst kann und darf sie nichts bedeuten wollen. Jenseits von diesem Geltungsbereich verliert sie jedes logische Daseinsrecht. Die Kräfte können an den Raumpunkten unmittelbar in Aktion treten, wie sie selbst nichts anderes als solche Relationen zwischen räumlichen und zeitlichen Verhältnissen ausdrücken. Dazu bedarf es nicht noch außerdem eines Kernes $a$, der die Forderung der Substanz in einer eignen Setzung zu vertreten und zu verantworten hätte. Der ausgedehnte Kern $a$ wird als eine Hypostasierung entlarvt. Es ist keine Erkenntnisbedingung, sondern nur ein leeres, unbekanntes Etwas; die bloße Forderung eines Beharrlichen, die von der Sinnlichkeit zu einem gegebenen, sinnlichen Substrat verdinglicht wird. Es ist der alte Fehler des Dogmatismus, der in der Vorwegnahme des Kerns $a$ sich in der Atomistik wiederholt. Gewiß ist es eine berechtigte Forderung des Denkens, ein festes, identisch verharrendes Substrat, einen letzten Beziehungspunkt der Aussàge zu haben. Aber der 
Dogmatismus verwechselt auch hier die Forderung mit der Erfüllung der Aufgabe. Den beharrlichen Komplex von Bestimmungen, den wir Ding nennen, betrachtet er, wie das naive Bewußtsein, als ein reines Datum und die relative Beständigkeit der Sinnendinge verleitet ihn dazu. Auf einer höheren Stufe der Reflexion enthüllen sich jedoch die sogenannten Dinge als das Allerunbeständigste, und für das wissenschaftliche Denken erwächst jetzt erst die Aufgabe, einen Bestand herzustellen, der den Wechsel der sinnlichen Gegenstände zu fester und beharrlicher Konstanz bringt.

Für das wissenschaftliche Denken gibt es keine absoluten Beziehungspunkte mehr, an die es seine Prädikationen heften könnte; das ewig sich Erhaltende gilt es nunmehr sich selbst zu erschaffen und das wahrhaft Dauernde aus eigner Kraft und Verantwortung hervorzubringen. Als das wirklich Konstante enthüllen sich einzig und allein die Relationen und Regeln des Denkens, in denen, aber freilich nur im unendlichen Fortgang, das Denken die schwankenden Erscheinungen befestigt. Das ist es, was dem Dogmatismus ewig verborgen bleibt, und dieser Irrtum ist so alt wie die Geschichte der Philosophie. Die Gebrechen des Aristotelischen Systems und seine Verkennung der Ideenlehre lassen sich aus diesem Grunde begreifen, wie denn auch seine Kategorienlehre hierin ihre Erklärung findet. ${ }^{14}$ ) Das Toranstellen der oüoía als der ersten Kategorie wurzelt in diesem Terlangen nach einem festen Bezugspunkt für die Aussagen im Crteil, der dann immer als ein gegebener antizipiert wird. Aristoteles abstrahiert die Kategorien als die allgemeinsten Merkmale der Gegenstände von den als gegeben vorausgesetzten Objekten, statt sie als konstruktive Bedingungen für den Aufbau der Wissenschaft abzuleiten. Die ouríx darf nicht die erste Kategorie sein, sie muß vielmehr in der Aristotelischen Bedeutung des róó $\tau$, des Einzeldings, die letzte werden. Die Atome aber sind in letzter Rücksicht doch nur Einzeldinge, und die endliche Ausdehnung, die bestimmte Gestalt ist nicht weniger ein Einzelnes. Daher wird die Kraft dem ausgerlehnten I)ing gegenüber zum logischen Prius, soIdeenlehre.

15) Vgl. bierzu besonders die beiden letzten Kapitel von Natorp, Platons 
forn die Kraft nicht ein mythisches Wesen, sondern das Gesetz, die Regel bedeutet, aus welcher der physische Gegenstand hervorwächst. In den Relationen der Kräfte muß das Objekt der Physik zur substantiellen Festigkeit gelangen und nicht in einem fertigen Ding zum Voraus gesetzt werden. „Was wir auch nur an der Materie kennen, sind lauter Verhältnisse, ... aber es sind darunter selbständige und beharrliche, dadurch uns ein bestimmter Gegenstand gegeben wird. $\mathrm{Da} B$ ich, wenn ich von diesen Verhältnissen abstrahiere, gar nichts weiter zu denken habe, hebt den Begriff von einem Dinge als Erscheinung nicht auf, auch nicht den Begriff von einem Gegenstande in abstracto, wohl aber alle Möglichkeit eines solchen, der nach bloßen Begriffen bestimmbar ist, d. i. eines Noumenon" (Kritik der reinen Vernunft, pag. 297).

Sofern also den Kraftrelationen oder der Atmosphäre $m$ im Ausdruck Faradays noch ein Substrat in Form eines endlichen, für das Denken nicht mehr. zerlegbaren Raumteiles untergelegt wird, ist der Relationscharakter der Denkfunktionen aufgehoben und ein absolutes Wesen postuliert. Damit verfällt das Atom dem Verdachte des Noumenon,.d. h. es wird zu einem Begriff, der durch das Gesetz der wissenschaftlichen Methodik ausgeschlossen ist.

\section{Substanz und Realität.}

Wenn somit die Substanz nicht in der Konstanz und Unbeweglichkeit der räumlichen Teile zu suchen ist, so darf sie doch auch nicht außer Beziehung auf die Gesetzlichkeit des Raumes gedacht werden. Der Gedanke der Substanz bedeutet die Forderung, für das veränderliche Geschehen in der Natur: eine feste, identisch sich erhaltende Grundlage żu finden. Diese Grundlage kann, wie wir gesehen haben, nicht die endliche Ausdehnung sein, sondern muß in der Identität einer gedanklichen Regel zum Vollzug kommen. Aber freilich muß sich diese Regel auf die räumlichen Gesetze beziehen; die als notwendige Grundlage zur Konstruktion des Naturobjektes anzuerkennen sind. Nur müssen die räumlichen Verhältnisse und Gestalten nicht als schon vorhanden, sondern aus ihren Elementen entspringend gedacht werden, d. h. die Substanz darf nicht als ruhendes Sein gefaßt werden, sondern als ein Entstehendes, 
Werdendes aus dem Denken erst entspringen: sie muß somit das Moment der Veränderung in sich aufnehmen.

Das universale Instrument, mit dessen Hilfe das Geschehen als ein Entstehendes, in der Veränderung Entspringendes, zur gesetzlichen Bestimmtheit kommen kann, ist die Methode der Infinitesimalanalysis. ${ }^{15}$ ) Die Analysis des Unendlichen gibt uns das Mittel, das Kontinuum des Raumes begrifflich zu beherrschen. Ohne $\mathrm{Zu}$ hilfenahme der Infinitesimalrechnung erscheint das Verfahren der endlichen Zahlen immer wie ein Operieren an einem gegebenen Stoff. Die Zahl macht Einschnitte in das zugrundeliegende Kontinuum, sie trifft Einteilungen und setzt Grenzen. Aber die Einheit, mit der sie zählt, ist unbestimmt und willkürlich, und ihre eigene Verfahrungsweise vermag das Rätsel nicht zur Lösung zu bringen, denn je tiefer sie in der Teilung hinabsteigen mag, es liegt in den Grenzen ihrer Methodik, daß sie das Problem damit nicht zu erschöpfen imstande ist, sondern sich an einen Fortgang ins Unendliche verwiesen sieht, von dem sich keine Bewältigung des Problems erwarten läßt. Es ist in der Definition des quantitativen Verfahrens begriffen, daß die Setzung von Quanten immer eine Setzung von Grenzen bedeutet, damit aber ein Zwischen, ein Kontinuum, schon voraussetzt. Das „Zwischen“ bedarf daher eines andern fundamentaleren Denkmittels, um begrifflich faßbar zu werden. Die Größe, das Quantum bleibt selbst unbestimmt, sofern nicht das Fundament, gleichsam das "Was" der Größe definiert ist. Dieses Fundament zur Erzeugung zu bringen, bedarf es einer andern Einheit, als welche die extensive Größe herzugeben vermag. Wir bezeichnen sie mit Leibniz, dem Entdecker des neuen Denkmittels, als intensive Größe. Ob zwar das Intensive nicht im eigentlichen Sinne eine Größe, sondern ein Verfahren, eine Regel des Denkens ist, so bleibt sie doch schlechterdings nur auf Größen bezogen. Sie ist eine neue Art Einheit, die, selbst kein Quantum, durch und in Operationen an Quanten zum Vollzug kommt, und so tritt sie der einzelnen, diskreten Setzung als das Gesetz des quantitativen Verfahrens selbst

15) Vergl. Cohen: Das Prinzip der Infinitesimalmethode, sowie: Logik der reinen Erkenntnis p. $102 \mathrm{ff}$. 
gegenüber, indem sie in einem universellen Verfahren die Allheit und Totalität der nach einem bestimmten Gesetz zu erzeugenden Quanta umfaßt. Dadurch bringt sie das endliche Quantum selbst zur Erzeugung, in einem radikaleren Sinne, als die Zusammensetzung aus diskreten Einheiten es vermag. Jede endliche Größe ist nunmehr nicht bloß eine isolierte, vereinzelte Setzung, sondern gleichsam die Grenze, das Ergebnis, das erst durch den ganzen Prozeß seinen bestimmten Ausdruck erhält; dadurch wird die diskrete Quantität selbst eine Spiegelung des Gंesetzes der Größensetzung, indem jeder Grenzpunkt nur durch den Verfluß und zugleich durch die ganze Fortsetzung des kontinuierlichen Größengebildes definiert ist. Damit gelingt es, im Punkte selbst den Verlauf des Geschehens auszudrücken; denn der unausgedehnte Punkt wird zum Ursprung, aus dem die Ausdehnung ausstrahlt. Die im Punkt fixierte Bestimmtheit ist demnach das eigentliche Reale, weil Radikale, das echte Fundament, aus dem die Größe sich aufbauen muß. Daher muß auch die Substanz dies Reale in sich aufnehmen, wenn anders sie die objektive Natur rechtmäßig vertreten will.

\section{Atome und Kraftzentra.}

Die punktuelle Bestimmtheit, die zugleich das Gesetz des weiteren Verlaufs des physischen Geschehens verbürgt, ist nun gerade das, was durch den Ausdruck "Kraft" bezeichnet wird. Und ließe sich an solchen intensiven Krafteinheiten der Gedanke der Erhaltung in Vollzug setzen, so wäre damit die Forderung der Substanz hinreichend befriedigt. Daher ist auch für Faraday nicht mehr die ausgedehnte Partikel das Substrat der Krậfte; die konstante Ausdehnung ist dem Substanzgedanken nicht wesentlich, sie verschwindet ganz oder zieht sich vielmehr in einem Punkt zusammen und wird damit zu einem bloßen Intensiven, einem Kraftzentrum. Und mit der Ausdehnung verschwindet natürlich auch die bestimmte Gestalt, denn auch Gestalt und Konfiguration finden in der punktuellen Gesetzlichkeit des Kraftzentrums ihre solidere Grundlage. „Auch hinsichtlich der Gestalt der Atome und ihres bestimmten und unveränderlichen Charakters muß man nun zu einer andern Auffassung gelangen. Ein Atom an und für sich kann man sich als 
kuglig oder sphäroidal vorstellen, oder wenn mehrere sich in allen Richtungen berührën, so kann man sie sich unter der Gestalt eines Dodekaëders denken, denn jedes muß an verschiedenen Seiten von zwölf andern umgeben sein und ihnen anliegen". (Bd. II, p. 262.) Denkt man sich jedoch ein Atom als Kraftzentrum, so muß das, was man gewöhnlich unter dem Ausdruck Gestalt versteht, als die Verteilung und relative Intensität der Kräfte aufgefaßt werden." Damit sieht sich Faraday auf die Annahme von Kraftzentren geführt, durch die er die materiellen Atome ersetzen will. "Ich weiß wohl, daß die Erscheinungen der Kristallisation der Chemie und Physik im allgemeinen unser Denken mächtig zur Vorstellung der Kraftzentren hinziehen. Ich selbst sehe mich einstweilen hypothetisch zur Annahme derselben genötigt und kann ohne sie nicht auskommen; dagegen erblicke ich in dem Begriff von materiellen Atomen, welche in festen, flüssigen und gasförmigen Körpern mehr oder weniger von einander abstehen, und deren $Z$ wischenräume nicht von Atomen eingenommen sein sollen, große Schwierigkeiten und finde in den aus dieser Vorstellung sich ergebenden Folgerungen bedeutende Widersprüche." (Bd. II p. 260.)

Indessen beruft sich Faraday bei der Diskussion des Atombegriffes und seines Verhältnisses zu den Kraftzentren auf das Werk des Jesuitenpaters Boscovich, und es ist, wie schon bemerkt wurde, keine geringe Schwierigkeit, die hierdurch für die Feststellung der eigentlichen Meinung Faradays entsteht. Denn die Theorie des Boscovich trägt wesentlich andre Züge, als die ihr von Faraday zugeschrieben werden. Es mag daher angebracht erscheinen, etwas näher auf das Verhältnis der beiden Auffassungen einzugehen, um durch Hervorhebung der Übereinstimmung und der Unterschiede beider Anschauungsweisen die Ansicht Faradays eindringlicher zu beleuchten. Wir setzen zunächst eine Stelle her, in der Faraday selbst den Lnterschied der Boscovichschen Theorie und der gewöhnlichen Atomistik erläutert. „Ehe ich diese Spekulation beschließe, will ich auf einige der wichtigen Lnterschiede hinweisen zwischen der Annahme von Atomen als bloßen Kraftzentren, wie diejenigen von Boscovich, und der Annahme von Molekülen eines materiellen Etwas, welche mit Kräften begabt und von ihnen umgeben sind. 
Nach der letzteren Auffassung besteht eine materielle Masse aus Atomen und Zwischenrïumen, nach der ersteren ist Materie überall gegenwärtig und es gibt keinen Raum, der nicht von ihr eingenommen wäre. In den Gasen berühren sich die Atome in Wahrheit ebenso wie in festen Körpern. Die Atome des Wassers berühren sich also, diese Substanz mag in Form : von Eis, Wasser oder. Dampf existieren; es gibt 'keinen leeren Zwischenraum. Ohne Zweifel variiert der Abstand der Kraftzentra von einander, aber das, was in Wahrheit das Materielle eines Atoms ist, berührt das Materielle seines Nachbaratoms."

„Demnach ist die Materie durchaus kontinuierlich, und wir haben in einer.materiellen Masse eine Trennung ihrer Atome durch Zwischenräume nicht anzunehmen. Die die Zentra umgebenden Kräfte verleihen diesen die Eigenschaften materieller Atome und diese Eigenschaften wiederum kombinieren sich, wenn viele Zentra sich durch ihre vereinten Kräfte zu einer Massse gruppieren, zu den Eigenschaften der Materie. Bei dieser Auffassung verschwinden alle Widersprüche, die sich bei einer Betrachtung der elektrischen Leitung und Isolation ergeben." (Bd. II, p. 261.)

Dieser Rekurs auf Boscovich kann nun nicht schlechthin als Identifizierung und Ineinssetzung beider Theorien gemeint sein. Sollen daher Mißdeutungen vermieden werden, die den Tatbestand zu verrücken geeignet sein könnten, so dürfen wir einer vergleichenden Gegenüberstellung beider Theorien nicht ausweichen. Es erscheint somit geboten, auf die.Tendenz der Boscovichschen Atomtheorie ein wenig näher einzugehen, umsomehr, als sie den Typus für eine bestimmte Gestaltung der Atomistik abgegeben hat, die bis in die neueste Zeit für ähnliche Versuche wegweisend geworden ist. Anderérs̄eits hat eine Konfrontierung der Theorien von Faraday und Boscovich den Nutzen, die Motive beider Forscher in ihren harmonierenden und kontrastierenden Richtungen in eine hellere Beleuchtung zu setzen.

(Schluß folgt.) 